\title{
BERRY-ESSEEN BOUNDS FOR \\ COMBINATORIAL CENTRAL \\ LIMIT THEOREMS AND \\ PATTERN OCCURRENCES, USING ZERO AND SIZE BIASING
}

\author{
LARRY GOLDSTEIN, ${ }^{*}$ University of Southern California
}

\begin{abstract}
Berry-Esseen-type bounds to the normal, based on zero- and size-bias couplings, are derived using Stein's method. The zero biasing bounds are illustrated in an application to combinatorial central limit theorems in which the random permutation has either the uniform distribution or one that is constant over permutations with the same cycle type, with no fixed points. The size biasing bounds are applied to the occurrences of fixed, relatively ordered subsequences (such as rising sequences) in a random permutation, and to the occurrences of patterns, extreme values, and subgraphs in finite graphs.
\end{abstract}

Keywords: Smoothing inequality; Stein's method; permutation; graph

2000 Mathematics Subject Classification: Primary 60F05; 60C05

\section{Introduction}

Using Stein's method, we develop Berry-Esseen-type bounds for normal approximation, based on zero- and size-bias couplings. The results are applied to bound the proximity to the normal in combinatorial central limit theorems in which the random permutation has either a uniform distribution or one that is constant over permutations with the same cycle type, with no fixed points; to counting the number of occurrences of fixed, relatively ordered subsequences, such as rising sequences, in a random permutation; and to counting the number of occurrences of patterns, local extremes, and subgraphs in finite graphs.

Stein's method [26], [28] uses characterizing equations to obtain bounds on the error when approximating distributions by a given target. For the normal, $X \sim \mathcal{N}\left(\mu, \sigma^{2}\right)$ if and only if

$$
\mathrm{E}(X-\mu) f(X)=\sigma^{2} \mathrm{E} f^{\prime}(X)
$$

for all absolutely continuous $f$ such that $\mathrm{E}\left|f^{\prime}(X)\right|<\infty$ (see [27]). From such a characterizing equation, a difference or differential equation can be set up to bound the difference between the expectation of a test function $h$ when evaluated on a given variable $Y$ and then on the variable $X$ having the target distribution. For the normal, with $X$ having the same mean $\mu$ and variance $\sigma^{2}$ as $Y$, the characterizing equation (1.1) leads to the differential equation

$$
h((y-\mu) / \sigma)-N h=\sigma^{2} f^{\prime}(y)-(y-\mu) f(y),
$$

where $N h=\mathrm{E} h(Z)$ with $Z \sim \mathcal{N}(0,1)$, the standard normal mean of the test function $h$. The expectation of the left-hand side can be evaluated at $Y$ by calculating the expectation of the

Received 20 August 2004; revision received 19 January 2005.

* Postal address: Department of Mathematics, University of Southern California, 3620 Vermont Avenue, KAP 108, Los Angeles, CA 90089-2532, USA. Email address: larry@math.usc.edu 
right-hand side using the bounded solution $f$ of (1.2) for the given $h$. By this device, Stein's method can handle various kinds of dependence, through the use of coupling constructions.

We consider and compare two couplings of a given $Y$ to achieve normal bounds. First, for $Y$ with mean 0 and variance $\sigma^{2} \in(0, \infty)$, we say that $Y^{*}$ has the $Y$-zero-biased distribution if

$$
\mathrm{E} Y f(Y)=\sigma^{2} \mathrm{E} f^{\prime}\left(Y^{*}\right)
$$

for all absolutely continuous functions $f$ such that the expectations of both sides exist. This zero-bias transformation from $Y$ to $Y^{*}$ was introduced in [15], and it was shown there that $Y^{*}$ exists for every mean-zero $Y$ with finite variance. Similarly, for $Y$ nonnegative with finite mean $\mathrm{E} Y=\mu$, we say that $Y^{\mathrm{s}}$ has the $Y$-size-biased distribution if

$$
\mathrm{E} Y f(Y)=\mu \mathrm{E} f\left(Y^{\mathrm{s}}\right)
$$

for all $f$ for which the expectations of both sides exist. The size-biased distribution exists for any nonnegative $Y$ with finite mean, and was used for normal approximation in [17].

A coupling $\left(Y, Y^{*}\right)$, where $Y^{*}$ has the $Y$-zero-biased distribution, lends itself to use in the Stein equation (1.2) in the following way: by (1.3), with $\sigma^{2}=1$ say, we have

$$
\mathrm{E} h(Y)-N h=\mathrm{E}\left(f^{\prime}(Y)-Y f(Y)\right)=\mathrm{E}\left(f^{\prime}(Y)-f^{\prime}\left(Y^{*}\right)\right) .
$$

Therefore, the difference between $Y$ and the normal, as tested on $h$, equals the difference between $Y$ and $Y^{*}$, as tested on $f^{\prime}$. Additionally, as observed in [15] and seen directly from (1.5), $Y$ is normal if and only if $Y \stackrel{\mathrm{D}}{=} Y^{*}$, where $\stackrel{\text { D }}{=}$ denotes equality in distribution. It is therefore natural that the distance from $Y$ to the normal can be expressed in terms of distance from $Y$ to $Y^{*}$. Our Theorem 1.1 makes this statement precise, showing that the distance from the standardized $Y$ to the normal, as measured by $\delta$ in (1.6), below, depends on the distribution of $Y$ only through a bound on $\left|Y^{*}-Y\right|$. A similar phenomenon was seen in [14], where it was shown, for any variable $Y$ (with mean 0 and variance $\sigma^{2}$ ) and $X \sim \mathcal{N}\left(0, \sigma^{2}\right)$, that

$$
d_{\mathrm{W}}(Y, X) \leq 2 d_{\mathrm{W}}\left(Y, Y^{*}\right)
$$

with $d_{\mathrm{W}}$ the Wasserstein distance.

The use of size-bias couplings in the Stein equation in (4.14), (4.15), and subsequent calculations below depends on the following identity, which is applied in a less direct manner than is (1.5): for $Y \geq 0$ with mean $\mu$ and variance $\sigma^{2}$,

$$
\mathrm{E}(Y-\mu) f(Y)=\mu \mathrm{E}\left(f\left(Y^{\mathrm{s}}\right)-f(Y)\right) \quad \text { and, therefore, } \sigma^{2}=\mu \mathrm{E}\left(Y^{\mathrm{s}}-Y\right) .
$$

With $W=(Y-\mu) / \sigma$, many authors (e.g. [7], [8], [10], [19], [24], and [25]) have been successful in obtaining bounds on the distance

$$
\delta=\sup _{h \in \mathscr{H}}|\mathrm{E} h(W)-N h|
$$

to the normal, over classes of nonsmooth functions $\mathscr{H}$, using Stein's method. Here we take the smoothing inequality approach, following [25]. In particular, $\mathscr{H}$ is a class of measurable functions on the real line with the following properties.

(i) The functions $h \in \mathscr{H}$ are uniformly bounded in absolute value by a constant, which we take to be 1 without loss of generality. 
(ii) For any real numbers $c$ and $d$, and for any $h(x) \in \mathscr{H}$, the function $h(c x+d)$ is also in $\mathcal{H}$.

(iii) For any $\varepsilon>0$ and $h \in \mathscr{H}$, the functions $h_{\varepsilon}^{+}$and $h_{\varepsilon}^{-}$, where

$$
\begin{gathered}
h_{\varepsilon}^{+}(x)=\sup _{|y| \leq \varepsilon} h(x+y), \quad h_{\varepsilon}^{-}(x)=\inf _{|y| \leq \varepsilon} h(x+y), \\
\quad \text { and } \tilde{h}_{\varepsilon}(x):=h_{\varepsilon}^{+}(x)-h_{\varepsilon}^{-}(x),
\end{gathered}
$$

are also in $\mathscr{H}$, and

$$
\mathrm{E} \tilde{h}_{\varepsilon}(Z) \leq a \varepsilon
$$

for some constant $a$ that depends only on the class $\mathscr{H}$.

The collection of indicator functions of all half-lines, and that of indicator functions of all intervals, for example, each form a class $\mathcal{H}$ that satisfies (1.7) and (1.8) with $a=(2 / \pi)^{1 / 2}$ and $a=2(2 / \pi)^{1 / 2}$, respectively (see, e.g. [25]).

Since the bound on $\delta$ in Theorem 1.1 depends only the size of $\left|Y^{*}-Y\right|$, it may be computed without the need for the calculation of the variances of certain conditional expectations that arise in other versions of Stein's method, the calculation of $\left(\operatorname{var}\left(E\left(\left(Y^{\prime \prime}-Y^{\prime}\right)^{2} \mid Y^{\prime}\right)\right)\right)^{1 / 2}$ for the exchangeable pair method, or the calculation of the term (1.13) for the size-bias coupling studied here.

Theorem 1.1. Let $Y$ be a mean-zero random variable with variance $\sigma^{2} \in(0, \infty)$, and let $Y^{*}$ be defined on the same space, with the $Y$-zero-biased distribution. If $\left|Y^{*}-Y\right| \leq 2 B$ for some $B \leq \frac{1}{24} \sigma$ then, for $\delta$ as in (1.6) and $a$ as in (1.8),

$$
\delta \leq A(37+12 A+112 a)
$$

where $A=2 B / \sigma$. For indicator functions of all half-lines and the indicator functions of all intervals, by using $a=(2 / \pi)^{1 / 2}$ and $a=2(2 / \pi)^{1 / 2}$, we respectively find that

$$
\delta \leq A(127+12 A) \text { and } \delta \leq A(216+12 A)
$$

See (4.22) and (4.23), below, for some variations on the bounds (1.9) and (1.12), respectively. We note that Theorem 1.1 immediately provides a bound on $\delta$ of order $\sigma^{-1}$ whenever $\left|Y^{*}-Y\right|$ is bounded. In Section 2, we apply Theorem 1.1 to random variables of the form

$$
Y=\sum_{i=1}^{n} a_{i, \pi(i)},
$$

depending on a fixed array of real numbers $\left\{a_{i j}\right\}_{i, j=1}^{n}$ and a random permutation $\pi \in \wp_{n}$, the symmetric group of order $n$. In Section 2.1, we consider $\pi$ to have the uniform distribution on $\delta_{n}$ and, in Section 2.2, we consider distributions constant with respect to cycle type, with no fixed points (conditions (2.10) and (2.12), respectively).

For a size-bias coupling $\left(Y, Y^{\mathrm{s}}\right)$, Theorem 1.2 gives a bound on $\delta$ that depends on the size of $\left|Y^{\mathrm{s}}-Y\right|$ and, additionally, on $\Delta$ in (1.13). While $\Delta$ may be difficult to calculate precisely in many cases, size-bias couplings can be more easily constructed for a broader range of examples than can the zero-bias couplings. 
Theorem 1.2. Let $Y \geq 0$ be a random variable with mean $\mu$ and variance $\sigma^{2} \in(0, \infty)$, and let $Y^{\mathrm{s}}$ be defined on the same space, with the $Y$-size-biased distribution. If $\left|Y^{\mathrm{s}}-Y\right| \leq B$ for some $B \leq \sigma^{3 / 2} /(6 \mu)^{1 / 2}$ then, for $\delta$ as in (1.6) and a as in (1.8),

$$
\delta \leq \frac{a A}{2}+\frac{\mu}{\sigma}\left((19+56 a) A^{2}+4 A^{3}\right)+\frac{23 \mu \Delta}{\sigma^{2}},
$$

where

$$
\Delta=\sqrt{\operatorname{var}\left(\mathrm{E}\left(Y^{\mathrm{s}}-Y \mid Y\right)\right)}
$$

and $A=B / \sigma$. For indicator functions of all half-lines and the indicator functions of all intervals, by using $a=(2 / \pi)^{1 / 2}$ and $a=2(2 / \pi)^{1 / 2}$, we respectively find that

$$
\delta \leq 0.4 A+\frac{\mu}{\sigma}\left(64 A^{2}+4 A^{3}\right)+\frac{23 \Delta \mu}{\sigma^{2}} \text { and } \delta \leq 0.8 A+\frac{\mu}{\sigma}\left(109 A^{2}+4 A^{3}\right)+\frac{23 \Delta \mu}{\sigma^{2}} .
$$

If the mean $\mu$ is of order $\sigma^{2}, B$ is bounded, and $\Delta=O\left(\sigma^{-1}\right)$, then $\delta$ will have order $\sigma^{-1}$. The application of Theorem 1.2 to counting the occurrences of fixed, relatively ordered subsequences, such as rising sequences, in a random permutation, and to counting the occurrences of color patterns, local maxima, and subgraphs in finite graphs is illustrated in Section 3. The proofs of Theorems 1.2 and 1.1 are given in Section 4.

Nothing should be inferred from the fact that the zero-bias applications presented here involve global dependence and that the dependence in the examples used to illustrate the sizebias approach is local: the exchangeable pair coupling on which our zero-biased constructions are based can also be applied in cases of local dependence, and the size-bias approach was applied in [17] to variables having global dependence.

In both zero and size biasing, a finite sum $Y=\sum_{\alpha \in \mathcal{A}} X_{\alpha}$ of independent variables on a finite index set $\mathcal{A}$ is biased by choosing a summand at random and replacing it with its biased version. To describe the zero biasing coupling, let $\left\{X_{\alpha}\right\}_{\alpha \in \mathcal{A}}$ be a collection of mean-zero variables with finite variance, and let $I$ be an independent random index with distribution

$$
P(I=\alpha)=\frac{w_{\alpha}}{\sum_{\beta \in \mathcal{A}} w_{\beta}},
$$

where $w_{\alpha}=\operatorname{var}\left(X_{\alpha}\right)$. It was shown in [15] that replacing $X_{I}$ by a variable $X_{I}^{*}$ having the $X_{I}$-zero-biased distribution, independent of $\left\{X_{\alpha}, \alpha \neq I\right\}$, gives

$$
Y^{*}=Y-X_{I}+X_{I}^{*},
$$

a variable having the $Y$-zero-biased distribution. Hence, when a sum of many independent variables of the same order is coupled in this way to its zero-biased version, the magnitudes of $\left(Y^{*}-Y\right) / \operatorname{var}(Y)^{1 / 2}$ and, therefore, of distance measures such as $\delta$, are small.

The construction of the size-biased coupling in the independent case is similar. Let $\left\{X_{\alpha}\right\}_{\alpha \in \mathcal{A}}$ be a collection of nonnegative variables with finite mean. Then, with $I$ a random index, independent of all other variables, having distribution (1.14) with $w_{\alpha}=\mathrm{E} X_{\alpha}$, the replacement of $X_{I}$ by a variable $X_{I}^{\mathrm{s}}$ with the $X_{I}$-size-bias distribution, independent of the remaining variables, gives a variable with the $Y$-size-biased distribution.

Zero-biased couplings of $Y^{*}$ to a sum $Y$ of nonindependent variables $X_{1}, \ldots, X_{n}$ is presently not very well understood. A construction in the presence of the weak global dependence of simple random sampling was given in [15]. Based on a remark in [15], we here exploit 
a connection between the zero-bias coupling and the exchangeable pair $\left(Y^{\prime}, Y^{\prime \prime}\right)$ of [28], with distribution $\mathrm{d} P\left(y^{\prime}, y^{\prime \prime}\right)$ satisfying $\mathrm{E}\left(Y^{\prime \prime} \mid Y^{\prime}\right)=(1-\lambda) Y^{\prime}$ for some $\lambda \in(0,1]$; in particular, we make use of a pair $\left(Y^{\dagger}, Y^{\ddagger}\right)$ with distribution proportional to $\left(y^{\prime}-y^{\prime \prime}\right)^{2} \mathrm{~d} P\left(y^{\prime}, y^{\prime \prime}\right)$.

The construction of $Y$ and $Y^{\mathrm{s}}$ on a common space for the sum of nonindependent variables $X_{1}, \ldots, X_{n}$ is more direct, and was described in Lemma 2.1 of [17]; we choose a summand with probability proportional to its expectation, replace it by one from its size-biased distribution, and then adjust the remaining variables according to the conditional distribution, given the value of the newly chosen variable. This construction is applied in Section 3, and a 'squared' zero biasing form of it in Section 2.

The mappings of a distribution $Y$ to its zero-biased version $Y^{*}$ or its size-biased version $Y^{\mathrm{s}}$ are special cases of distributional transformations from $Y$ to some $Y^{(m)}$, which are specified by a function $H$ and the characterizing equation $\mathrm{E} H(Y) f(Y)=\eta \mathrm{E} f^{(m)}\left(Y^{(m)}\right)$ for all smooth $f$, where $f_{(m)}$ denotes the $m$ th derivative of $f$ and $\eta$ necessarily equals $(m !)^{-1} \mathrm{E} H(Y) Y^{m}$ when this expectation exists. The zero-bias and size-bias transformation correspond to $m=1$ and $H(x)=x$, and $m=0$ and $H(x)=x^{+}$, respectively. In general, such a $Y^{(m)}$ exists when $H$ and $Y$ satisfy certain sign-change and orthogonality properties, as discussed in [16].

\section{Zero biasing: combinatorial central limit theorems}

In this section, we illustrate the use of Theorem 1.1 to obtain Berry-Esseen bounds in combinatorial central limit theorems, that is for variables $Y$ as in (1.11). In Section 2.1, we do so for permutations having the uniform distribution over the symmetric group and, in Section 2.2, we do so for permutations with distribution constant over those with cycles of the same type and with no fixed points. We first present Proposition 2.1, which suggests a method for the construction of zero-bias couplings, based on the existence of exchangeable pairs; its statement appears in [15].

Proposition 2.1. Let $Y^{\prime}$ and $Y^{\prime \prime}$ be an exchangeable pair, with distribution $\mathrm{d} P\left(y^{\prime}, y^{\prime \prime}\right)$ and $\operatorname{var}\left(Y^{\prime}\right)=\sigma^{2} \in(0, \infty)$, that satisfies the linearity condition

$$
\mathrm{E}\left(Y^{\prime \prime} \mid Y^{\prime}\right)=(1-\lambda) Y^{\prime} \text { for some } \lambda \in(0,1] \text {. }
$$

Then

$$
\mathrm{E} Y^{\prime}=0 \text { and } \mathrm{E}\left(Y^{\prime}-Y^{\prime \prime}\right)^{2}=2 \lambda \sigma^{2} \text {, }
$$

and if $Y^{\dagger}$ and $Y^{\ddagger}$ have distribution

$$
\mathrm{d} P^{\dagger}\left(y^{\prime}, y^{\prime \prime}\right)=\frac{\left(y^{\prime}-y^{\prime \prime}\right)^{2}}{\mathrm{E}\left(Y^{\prime}-Y^{\prime \prime}\right)^{2}} \mathrm{~d} P\left(y^{\prime}, y^{\prime \prime}\right)
$$

and $U \sim \mathcal{U}[0,1]$ is independent of $Y^{\dagger}$ and $Y^{\ddagger}$, then the variable

$$
Y^{*}=U Y^{\dagger}+(1-U) Y^{\ddagger} \text { has the } Y^{\prime} \text {-zero-biased distribution. }
$$

Proof. The claims in (2.2) follow from (2.1) and exchangeability. Hence, we need only show that $Y^{*}$ in (2.4) satisfies (1.3). For a differentiable test function $f$,

$$
\begin{aligned}
\sigma^{2} \mathrm{E} f^{\prime}\left(U Y^{\dagger}+(1-U) Y^{\ddagger}\right) & =\sigma^{2} \mathrm{E}\left(\frac{f\left(Y^{\dagger}\right)-f\left(Y^{\ddagger}\right)}{Y^{\dagger}-Y^{\ddagger}}\right) \\
& =\sigma^{2} \mathrm{E}\left(\frac{\left(Y^{\prime}-Y^{\prime \prime}\right)\left(f\left(Y^{\prime}\right)-f\left(Y^{\prime \prime}\right)\right)}{\mathrm{E}\left(Y^{\prime}-Y^{\prime \prime}\right)^{2}}\right) .
\end{aligned}
$$


Now, if we use (2.1) to obtain $\mathrm{E} Y^{\prime \prime} f\left(Y^{\prime}\right)=(1-\lambda) \mathrm{E} Y^{\prime} f\left(Y^{\prime}\right)$, followed by (2.2), expanding yields

$$
\sigma^{2} \mathrm{E}\left(\frac{Y^{\prime} f\left(Y^{\prime}\right)-Y^{\prime \prime} f\left(Y^{\prime}\right)-Y^{\prime} f\left(Y^{\prime \prime}\right)+Y^{\prime \prime} f\left(Y^{\prime \prime}\right)}{\mathrm{E}\left(Y^{\prime}-Y^{\prime \prime}\right)^{2}}\right)=\frac{2 \lambda \sigma^{2} \mathrm{E} Y^{\prime} f\left(Y^{\prime}\right)}{\mathrm{E}\left(Y^{\prime}-Y^{\prime \prime}\right)^{2}}=\mathrm{E} Y^{\prime} f\left(Y^{\prime}\right) .
$$

Example 2.1. Given a mean-zero finite-variance $Y^{\prime}$, let $Y^{\prime \prime}$ be an independent copy of $Y^{\prime}$. The pair $\left(Y^{\prime}, Y^{\prime \prime}\right)$ satisfies the conditions of Proposition 2.1 with $\lambda=1$ and, hence, $Y^{*}$ as in (2.4) has the $Y^{\prime}$-zero-biased distribution with $\left(Y^{\dagger}, Y^{\dagger}\right)$ as in (2.3). However, coupling $Y^{\prime}$ close to $Y^{\prime \prime}$, so that $Y^{\dagger}$ is close to $Y^{\ddagger}$, causes $B$ and, therefore, the bound $\delta$ of Theorem 1.1 to be small.

Remark 2.1. The following construction of $\left(Y^{\dagger}, Y^{\dagger}\right)$, suggested by Proposition 2.1, is similar to the one used for size biasing (see Lemma 2.1 of [17] and Section 3). Given $Y^{\prime}$, first construct an exchangeable $Y^{\prime \prime}$ close to $Y^{\prime}$ satisfying (2.1), and then independently construct the variables appearing in the 'square-biased' term $\left(Y^{\prime}-Y^{\prime \prime}\right)^{2}$. Lastly, adjust the remaining variables that make up $\left(Y^{\prime}, Y^{\prime \prime}\right)$ to have their original conditional distribution, given the newly generated variables.

Example 2.2. Let $\left\{X_{i}^{\prime}, X_{i}^{\prime \prime}\right\}_{i=1, \ldots, n}$ be independent and identically distributed mean-zero variables with finite variance, let $Y^{\prime}=\sum_{i=1}^{n} X_{i}^{\prime}$, and let $I$ be an independent random index with the uniform distribution over $\{1, \ldots, n\}$. Letting $Y^{\prime \prime}=Y^{\prime}-X_{I}^{\prime}+X_{I}^{\prime \prime}$, we find that the pair $\left(Y^{\prime}, Y^{\prime \prime}\right)$ is exchangeable and satisfies the conditions of Proposition 2.1 with $\lambda=1 / n$. Set $S=$ $\sum_{i \neq I} X_{i}^{\prime}$ and $\left(T^{\prime}, T^{\prime \prime}\right)=\left(X_{I}^{\prime}, X_{I}^{\prime \prime}\right)$. Applying Example 2.1 to $\left(T^{\prime}, T^{\prime \prime}\right)$ and forming $\left(T^{\dagger}, T^{\ddagger}\right)$ independently of $\left\{X_{i}^{\prime}, X_{i}^{\prime \prime}\right\}_{i \neq I}$ gives $U T^{\dagger}+(1-U) T^{\ddagger}=X_{I}^{*}$. By their independence from $X_{I}^{\prime}$ and $X_{I}^{\prime \prime},\left\{X_{i}^{\prime}, X_{i}^{\prime \prime}\right\}_{i \neq I}$ already have their original conditional distribution, given $\left(T^{\dagger}, T^{\ddagger}\right)$; hence, $Y^{*}=S+X_{I}^{*}$ in agreement with (1.15).

Applying this construction in the presence of dependence results in $S$, a function of the variables that can be kept fixed, and variables $T^{\prime}, T^{\dagger}$, and $T^{\ddagger}$, on a joint space, such that

$$
Y^{\prime}=S+T^{\prime}, \quad Y^{\dagger}=S+T^{\dagger}, \quad \text { and } \quad Y^{\ddagger}=S+T^{\ddagger} .
$$

When $T^{\prime}, T^{\dagger}$, and $T^{\ddagger}$ are all bounded by $B,(2.4)$ gives

$$
\left|Y^{*}-Y^{\prime}\right|=\left|U T^{\dagger}+(1-U) T^{\ddagger}-T^{\prime}\right| \leq U\left|T^{\dagger}\right|+(1-U)\left|T^{\ddagger}\right|+\left|T^{\prime}\right| \leq 2 B .
$$

Let an array $\left\{a_{i j}\right\}_{i, j=1}^{n}$ of real numbers satisfy

$$
\sum_{j=1}^{n} a_{i j}=0 \quad \text { for all } i, \text { and set } \quad C=\max _{i, j}\left|a_{i j}\right| .
$$

By replacing $Y$ in (1.11) by $Y-\mathrm{E} Y$ we assume, without loss of generality, that $\mathrm{E} a_{i, \pi(i)}=0$ for every $i$. In Theorem 2.1, below, where $\pi$ is uniformly distributed over $8_{n}$, this assumption is equivalent to (2.7). In Theorem 2.2, since $\pi$ has no fixed points, by (2.12), without loss of generality we have $a_{i i}=0$ for all $i$ in (2.11). In addition, since the distribution of $\pi$ is constant on permutations having the same cycle type, by (2.10), $\mathrm{E} a_{i, \pi(i)}=(1 /(n-1)) \sum_{j \neq i} a_{i j}$ and the mean-zero assumption is again equivalent to (2.7). Avoiding trivial cases, we also assume that $\operatorname{var}(Y)=\sigma^{2}>0$. For ease of notation, we write $Y^{\prime}$ and $\pi^{\prime}$ interchangeably for $Y$ and $\pi$, respectively, in the remainder of this section. 
In Sections 2.1 and 2.2, the construction above produces variables $Y^{\prime}, Y^{\dagger}$, and $Y^{\ddagger}$ given by (1.11) (with $\pi$ replaced by $\pi^{\prime}, \pi^{\dagger}$, and $\pi^{\ddagger}$, respectively) and a set of indices $\mathcal{I}$ outside of which these permutations agree, such that (2.5) holds with

$$
S=\sum_{i \notin \mathcal{I}} a_{i, \pi^{\prime}(i)}, \quad T^{\prime}=\sum_{i \in \mathcal{I}} a_{i, \pi^{\prime}(i)}, \quad T^{\dagger}=\sum_{i \in \mathcal{I}} a_{i, \pi^{\dagger}(i)}, \quad \text { and } \quad T^{\ddagger}=\sum_{i \in \mathcal{I}} a_{i, \pi^{\ddagger}(i)} .
$$

Therefore, $B$ in (2.6) can be set equal to $C$ in (2.7) times a worst-case bound on the size of $\mathcal{L}$. The specifications of $\pi^{\prime}, \pi^{\dagger}$, and $\pi^{\ddagger}$ are given in terms of transpositions $\tau_{i j}$, those permutations satisfying $\tau_{i j}(i)=j, \tau_{i j}(j)=i$, and $\tau_{i j}(k)=k$ for all $k \notin\{i, j\}$.

\subsection{Uniform permutation distribution}

Many authors (see, e.g. [7], [20], and [29]) have considered the normal approximation to the distribution of (1.11) when $\pi$ is a permutation chosen uniformly from $\wp_{n}$. In Theorem 2.1, the dependence of $\delta$ on $C$ is not as refined as the bound in [7], which depends on an unspecified universal constant times the normalized absolute third moments of the $\left\{a_{i j}\right\}_{i, j=1}^{n}$. Here, on the other hand, an explicit constant is provided.

Theorem 2.1. With $n \geq 3$, let $\left\{a_{i j}\right\}_{i, j=1}^{n}$ satisfy (2.7) and let $\pi$ be a random permutation with the uniform distribution over $8_{n}$. Then, with $C$ as in (2.7), conclusions (1.9) and (1.10) of Theorem 1.1 hold for the sum $Y=\sum_{i=1}^{n} a_{i, \pi(i)}$, with $A=8 C / \sigma$ when $A \leq \frac{1}{12}$.

Proof. Given $\pi^{\prime}$, take $(I, J)$ to be independent of $\pi^{\prime}$, uniformly over all pairs with $1 \leq I \neq$ $J \leq n$, and set $\pi^{\prime \prime}=\pi^{\prime} \tau_{I, J}$. In particular, $\pi^{\prime \prime}(i)=\pi^{\prime}(i)$ for $i \notin\{I, J\}$; the variables $Y^{\prime}$ and $Y^{\prime \prime}$, given by (1.11) with permutations $\pi^{\prime}$ and $\pi^{\prime \prime}$, respectively, are exchangeable; and

$$
Y^{\prime}-Y^{\prime \prime}=\left(a_{I, \pi^{\prime}(I)}+a_{J, \pi^{\prime}(J)}\right)-\left(a_{I, \pi^{\prime}(J)}+a_{J, \pi^{\prime}(I)}\right) .
$$

The linearity condition (2.1) is satisfied, with $\lambda=2 /(n-1)$, since, from (2.9) and (2.7),

$$
\begin{aligned}
\mathrm{E}\left(Y^{\prime}-Y^{\prime \prime} \mid \pi^{\prime}\right) & =2\left(\frac{1}{n} \sum_{i=1}^{n} a_{i, \pi^{\prime}(i)}-\frac{1}{n(n-1)} \sum_{i \neq j} a_{i, \pi^{\prime}(j)}\right) \\
& =2\left(\frac{1}{n} \sum_{i=1}^{n} a_{i, \pi^{\prime}(i)}+\frac{1}{n(n-1)} \sum_{i=1}^{n} a_{i, \pi^{\prime}(i)}\right)=\frac{2}{n-1} Y^{\prime} .
\end{aligned}
$$

To construct $\left(Y^{\dagger}, Y^{\ddagger}\right)$ with distribution proportional to $\left(y^{\prime}-y^{\prime \prime}\right)^{2} \mathrm{~d} P\left(y^{\prime}, y^{\prime \prime}\right)$, choose $I^{\dagger}$, $K^{\dagger}, J^{\dagger}$, and $L^{\dagger}$ with distribution proportional to the squared difference $\left(Y^{\prime}-Y^{\prime \prime}\right)^{2}$, that is

$$
\mathrm{P}\left(I^{\dagger}=i, K^{\dagger}=k, J^{\dagger}=j, L^{\dagger}=l\right) \sim\left[\left(a_{i j}+a_{j l}\right)-\left(a_{i l}+a_{j k}\right)\right]^{2},
$$

let

$$
\pi^{\dagger}= \begin{cases}\pi \tau_{\pi^{-1}\left(K^{\dagger}\right), J^{\dagger}} & \text { if } L^{\dagger}=\pi\left(I^{\dagger}\right) \text { and } K^{\dagger} \neq \pi\left(J^{\dagger}\right), \\ \pi \tau_{\pi^{-1}\left(L^{\dagger}\right), I^{\dagger}} & \text { if } L^{\dagger} \neq \pi\left(I^{\dagger}\right) \text { and } K^{\dagger}=\pi\left(J^{\dagger}\right), \\ \pi \tau_{\pi^{-1}\left(K^{\dagger}\right), I^{\dagger}} \tau_{\pi^{-1}\left(L^{\dagger}\right), J^{\dagger}} & \text { otherwise, }\end{cases}
$$

and let $\pi^{\dagger}=\pi^{\dagger} \tau_{I^{\dagger}, J^{\dagger}}$. Then (2.5) and (2.8) hold with $\mathcal{L}=\left\{I^{\dagger}, \pi^{-1}\left(K^{\dagger}\right), \pi^{-1}\left(L^{\dagger}\right)\right\}$, a set of size at most four. Hence, by (2.6), $\left|Y^{*}-Y\right| \leq 8 C$. 


\subsection{Permutations with distribution constant over cycle type}

In this section, we focus on the normal approximation of $Y$ (as in (1.11)) when the distribution of the random permutation $\pi$ is a function only of its cycle type. Our framework includes the case considered in [22], i.e. the uniform distribution over permutations with a single cycle.

Consider a permutation $\pi \in \wp_{n}$ represented in cycle form: in $\varsigma_{7}$, for example, $\pi=$ $((1,3,7,5),(2,6,4))$ is the permutation consisting of one 4-cycle in which $1 \rightarrow 3 \rightarrow 7 \rightarrow$ $5 \rightarrow 1$ and one 3 -cycle where $2 \rightarrow 6 \rightarrow 4 \rightarrow 2$. For $q=1, \ldots, n$, let $c_{q}(\pi)$ be the number of $q$-cycles of $\pi$. We say that the permutations $\pi$ and $\sigma$ are of the same cycle type if $c_{q}(\pi)=c_{q}(\sigma)$ for all $q=1, \ldots, n ; \pi$ and $\sigma$ are of the same cycle type if and only if $\pi$ and $\sigma$ are conjugate, i.e. if and only if there exists a permutation $\rho$ such that $\pi=\rho^{-1} \sigma \rho$. Hence, we say a probability measure $\mathrm{P}$ on $\delta_{n}$ is constant over cycle type if

$$
\mathrm{P}(\pi)=\mathrm{P}\left(\rho^{-1} \pi \rho\right) \text { for all } \pi, \rho \in \varsigma_{n} .
$$

In [18], the authors considered a statistical test for determining when a given pairing of $n=2 m$ observations shows an unusually high level of similarity. The test statistic is of the form (1.11) and, under the null hypothesis of no distinguished pairing, the distribution $\mathrm{P}$ satisfies (2.10) with $\mathrm{P}(\pi)$ equal to a constant if $\pi$ has $m$ 2-cycles and $\mathrm{P}(\pi)=0$ otherwise; that is, under the null hypothesis, $\mathrm{P}$ is uniform over permutations with $m$ 2-cycles. Bounds between the normal and the null distribution of $Y$ were determined in [18] using a construction in which an exchangeable $\pi^{\prime \prime}$ is obtained from $\pi$ by a transformation that preserves the $m$ 2-cycle structure. The construction in Theorem 2.2 preserves the cycle structure in general and, when there are $m$ 2-cycles, specializes to one similar, but not equivalent, to that of [18].

Theorem 2.2. With $n \geq 4$, let an array $\left\{a_{i j}\right\}_{i j=1}^{n}$ of real numbers satisfy (2.7), let

$$
a_{i j}=a_{j i} \quad \text { and } \quad a_{i i}=0
$$

and let $\pi \in \wp_{n}$ be a random permutation with distribution $\mathrm{P}$ constant on cycle type, with no fixed points. That is, $\mathrm{P}$ satisfies (2.10), (2.11), and

$$
\mathrm{P}(\pi)=0 \quad \text { if } c_{1}(\pi) \neq 0 .
$$

Then, with $C$ as in (2.7), conclusions (1.9) and (1.10) of Theorem 1.1 hold for the sum $Y=$ $\sum_{i=1}^{n} a_{i, \pi(i)}$, with $A=40 C / \sigma$ when $A \leq \frac{1}{12}$.

Proof. To fully highlight the reason for the imposition of the conditions (2.11) and (2.12), and also to make the complete case analysis easier to follow, we initially consider an array satisfying only the consequence $\sum_{1 \leq i, j \leq n} a_{i j}=0$ of (2.7), and a P not necessarily satisfying (2.12).

Again, using the construction outlined in Remark 2.1, we first construct $\pi^{\prime \prime}$ from the given $\pi^{\prime}$. Let $I$ and $J, 1 \leq I \neq J \leq n$, be chosen uniformly and independently of $\pi^{\prime}$, and let $\pi^{\prime \prime}=\tau_{I J} \pi^{\prime} \tau_{I J}$; that is, $\pi^{\prime \prime}$ is obtained by interchanging $I$ and $J$ in the cycle representation of $\pi^{\prime}$. We claim that the pair $\left(\pi^{\prime}, \pi^{\prime \prime}\right)$ is exchangeable. For fixed permutations $\sigma^{\prime \prime}$ and $\sigma^{\prime}$, if $\sigma^{\prime} \neq \tau_{I J} \sigma^{\prime \prime} \tau_{I J}$ then

$$
\mathrm{P}\left(\pi^{\prime \prime}=\sigma^{\prime \prime}, \pi^{\prime}=\sigma^{\prime}\right)=0=\mathrm{P}\left(\pi^{\prime}=\sigma^{\prime \prime}, \pi^{\prime \prime}=\sigma^{\prime}\right) .
$$

Otherwise, $\sigma^{\prime}=\tau_{I J} \sigma^{\prime \prime} \tau_{I J}$ and, using (2.10) in the second equality, we have

$$
\begin{aligned}
\mathrm{P}\left(\pi^{\prime \prime}=\sigma^{\prime \prime}, \pi^{\prime}=\sigma^{\prime}\right) & =\mathrm{P}\left(\pi^{\prime}=\sigma^{\prime}\right)=\mathrm{P}\left(\pi^{\prime}=\tau_{I J} \sigma^{\prime} \tau_{I J}\right) \\
& =\mathrm{P}\left(\pi^{\prime \prime}=\sigma^{\prime}\right)=\mathrm{P}\left(\pi^{\prime}=\sigma^{\prime \prime}, \pi^{\prime \prime}=\sigma^{\prime}\right) .
\end{aligned}
$$


Consequently, $Y$ and $Y^{\prime \prime}$, given by (1.11) with permutations $\pi$ and $\pi^{\prime \prime}$, respectively, are exchangeable. By conditioning on $\pi$, we can show that $\left(Y^{\prime}, Y^{\prime \prime}\right)$ satisfies the linearity condition (2.1) with $\lambda=4 / n$.

Let $S$ be the size of the set $\{I, J, \pi(I), \pi(J)\}$ and, for $i \in\{1, \ldots, n\}$, let $|i|$ denote the number of elements in the cycle of $\pi$ that contains $i$. Since $I \neq J$, we have $2 \leq S \leq 4$. When $S=2$, either $\pi(I)=I$ and $\pi(J)=J$, or $\pi(I)=J$ and $\pi(J)=I$; in both cases, $\pi^{\prime \prime}=\pi$. There are four cases for $S=3$ : either $A_{I, J}=\{|I|=1,|J| \geq 2\}$ or $I$ and $J$ are interchanged (denoted by $A_{J, I}$ ); or $I, J$, and $\pi(J)$ are three consecutive, distinct values of $\pi$, indicated by $B_{I, J}$, or $I$ and $J$ are interchanged (denoted by $B_{J, I}$ ). The case $S=4$ is indicated by $F$. Hence,

$$
\begin{aligned}
Y^{\prime}-Y^{\prime \prime}= & \left(a_{I, I}+a_{\pi^{-1}(J), J}+a_{J, \pi(J)}-\left(a_{J, J}+a_{\pi^{-1}(J), I}+a_{I, \pi(J)}\right)\right) A_{I, J} \\
& +\left(a_{J, J}+a_{\pi^{-1}(I), I}+a_{I, \pi(I)}-\left(a_{I, I}+a_{\pi^{-1}(I), J}+a_{J, \pi(I)}\right)\right) A_{J, I} \\
& +\left(a_{\pi^{-1}(I), I}+a_{I, J}+a_{J, \pi(J)}-\left(a_{\pi^{-1}(I), J}+a_{J, I}+a_{I, \pi(J)}\right)\right) B_{I, J} \\
& +\left(a_{\pi^{-1}(J), J}+a_{J, I}+a_{I, \pi(I)}-\left(a_{\pi^{-1}(J), I}+a_{I, J}+a_{J, \pi(I)}\right)\right) B_{J, I} \\
& +\left(a_{\pi^{-1}(I), I}+a_{I, \pi(I)}+a_{\pi^{-1}(J), J}+a_{J, \pi(J)}\right. \\
& \left.-\left(a_{\pi^{-1}(I), J}+a_{J, \pi(I)}+a_{\pi^{-1}(J), I}+a_{I, \pi(J)}\right)\right) F .
\end{aligned}
$$

For example, using the fact that the sum of $a_{\pi^{-1}(J), J}$ is the same as that of $a_{J, \pi(J)}$ over a given cycle, the contribution to $n(n-1) \mathrm{E}\left(Y^{\prime}-Y^{\prime \prime} \mid \pi\right)$ from $A_{I, J}=\{|I|=1,|J| \geq 2\}$, added to the equal one from $A_{J, I}$, simplifies to

$$
\begin{aligned}
2\left(n-3 c_{1}(\pi)\right) \sum_{|i|=1} a_{i, i}+4 c_{1}(\pi) \sum_{|i| \geq 1} a_{i, \pi(i)} & -2 c_{1}(\pi) \sum_{|i| \geq 2} a_{i, i} \\
& -2 \sum_{|i|=1,|j| \geq 2} a_{i, j}-2 \sum_{|i| \geq 2,|j|=1} a_{i, j} .
\end{aligned}
$$

Next, the equal contributions from $B_{I, J}=\mathbf{1}(\pi(I)=J,|I| \geq 3)$ and $B_{J, I}$ sum to

$$
6 \sum_{|i| \geq 3} a_{i, \pi(i)}-4 \sum_{|i| \geq 3} a_{\pi^{-1}(i), \pi(i)}-2 \sum_{|i| \geq 3} a_{\pi(i), i} .
$$

On $F=\mathbf{1}(|I| \geq 2,|J| \geq 2, I \neq J, \pi(I) \neq J, \pi(J) \neq I)$, the contribution from $a_{\pi^{-1}(I), I}$ is

$$
\sum_{|i|,|j| \geq 2} a_{\pi^{-1}(i), i} \mathbf{1}(i \neq j, \pi(i) \neq j, \pi(j) \neq i) .
$$

Let $i \cong j$ denote the fact that $i$ and $j$ are elements of the same cycle. When $i \cong j$ and $\{i, j, \pi(i), \pi(j)\}$ are distinct, we have $|i| \geq 4$ and there are $|i|-3$ possible choices for $j \cong i$ that satisfy the conditions in the indicator function in (2.16). Hence, the case $i \cong j$ contributes

$$
\begin{aligned}
\sum_{|i| \geq 4} a_{\pi^{-1}(i), i} \sum_{j \cong i} \mathbf{1}(i \neq j, \pi(i) \neq j, \pi(j) \neq i) & =\sum_{|i| \geq 4} a_{\pi^{-1}(i), i}(|i|-3) \\
& =\sum_{|i| \geq 3}(|i|-3) a_{i, \pi(i)}
\end{aligned}
$$


When $i \not j$ the conditions in the indicator function (2.16) are satisfied if and only if $|i| \geq 2$ and $|j| \geq 2$. For $|i| \geq 2$, there are $n-|i|-c_{1}(\pi)$ choices for $j$, so the case $i \neq j$ contributes

$$
\sum_{|i| \geq 2} a_{\pi^{-1}(i), i} \sum_{j \neq i,|j| \geq 2} 1=\sum_{|i| \geq 2}\left(n-|i|-c_{1}(\pi)\right) a_{i, \pi(i)} .
$$

The next three terms of $F$ give the same as the first, so in total we have

$$
4\left(n-2-c_{1}(\pi)\right) \sum_{|i|=2} a_{i, \pi(i)}+4\left(n-3-c_{1}(\pi)\right) \sum_{|i| \geq 3} a_{i, \pi(i)} .
$$

Decomposing the contribution from the fifth term according to whether $i \cong j$ or $i \not j$ gives

$$
\begin{aligned}
& -\sum_{|i|,|j| \geq 2} a_{\pi^{-1}(i), j} \mathbf{1}(i \neq j, \pi(i) \neq j, \pi(j) \neq i) \\
& \quad-\sum_{|i| \geq 4} \sum_{j \cong i} a_{\pi^{-1}(i), j} \mathbf{1}(i \neq j, \pi(i) \neq j, \pi(j) \neq i)-\sum_{|i|,|j| \geq 2} \sum_{j \neq i} a_{\pi^{-1}(i), j} \\
& =-\sum_{|i| \geq 4} \sum_{j \cong i} a_{\pi^{-1}(i), j}+\sum_{|i| \geq 4}\left(a_{\pi^{-1}(i), i}+a_{\pi^{-1}(i), \pi(i)}+a_{\pi^{-1}(i), \pi^{-1}(i)}\right)-\sum_{|i|,|j| \geq 2} \sum_{j \neq i} a_{i, j} \\
& =-\sum_{|i| \geq 4} \sum_{j \cong i} a_{i, j}+\sum_{|i| \geq 4}\left(a_{i, \pi(i)}+a_{\pi^{-1}(i), \pi(i)}+a_{i, i}\right)-\sum_{|i|,|j| \geq 2} \sum_{j \neq i} a_{i, j} .
\end{aligned}
$$

To simplify (2.18), let $a \wedge b=\min (a, b)$ and consider the decomposition

$$
\sum_{i, j=1}^{n} a_{i, j}=\sum_{|i| \geq 4} \sum_{j \cong i} a_{i, j}+\sum_{|i| \leq 3} \sum_{j \cong i} a_{i, j}+\sum_{|i|,|j| \geq 2} \sum_{j \neq i} a_{i, j}+\sum_{|i| \wedge|j|=1} \sum_{j \nsucceq i} a_{i, j} .
$$

Since $\sum_{i, j} a_{i j}=0$, we may replace the sum of the first and last terms in (2.18) by the sum of the second and fourth terms on the right-hand side of (2.19), resulting in

$$
\begin{aligned}
\sum_{|i| \leq 3} & \sum_{j \cong i} a_{i, j}+\sum_{|i| \wedge|j|=1} \sum_{j \nsubseteq i} a_{i, j}+\sum_{|i| \geq 4}\left(a_{i, \pi(i)}+a_{\pi^{-1}(i), \pi(i)}+a_{i, i}\right) \\
& =\sum_{|i| \leq 2} \sum_{j \cong i} a_{i, j}+\sum_{|i| \wedge|j|=1} \sum_{j \neq i} a_{i, j}+\sum_{|i| \geq 3}\left(a_{i, \pi(i)}+a_{\pi^{-1}(i), \pi(i)}+a_{i, i}\right),
\end{aligned}
$$

where we have used the fact that $\pi^{2}(i)=\pi^{-1}(i)$ when $|i|=3$. Similarly shifting the $|i|=2$ term, we obtain

$$
\begin{array}{r}
\sum_{|i|=1} a_{i, i}+\sum_{|i| \wedge|j|=1} \sum_{j \neq i} a_{i, j}+\sum_{|i| \geq 2}\left(a_{i, \pi}(i)+a_{i, i}\right)+\sum_{|i| \geq 3} a_{\pi^{-1}(i), \pi(i)} \\
=\sum_{|i| \wedge|j|=1} \sum_{j \neq i} a_{i, j}+\sum_{|i| \geq 2} a_{i, \pi(i)}+\sum_{|i| \geq 1} a_{i, i}+\sum_{|i| \geq 3} a_{\pi^{-1}(i), \pi(i)} .
\end{array}
$$

Combining this with the next three terms of $F$, each of which yields the same contribution, gives

$$
4 \sum_{|i| \geq 2} a_{i, \pi(i)}+4 \sum_{|i| \geq 3} a_{\pi^{-1}(i), \pi(i)}+4 \sum_{|i| \geq 1} a_{i, i}+4 \sum_{|i| \wedge|j|=1} \sum_{j \neq i} a_{i, j} .
$$


Combining (2.20) with the contribution (2.17) of the first four terms of $F$, the $A_{I, J}$ and $A_{J, I}$ terms in (2.14), and the $B_{I, J}$ and $B_{J, I}$ terms in (2.15), yields $n(n-1) \mathrm{E}\left(Y^{\prime}-Y^{\prime \prime} \mid \pi^{\prime}\right)$; after cancelling the terms involving $a_{\pi^{-1}(i), \pi(i)}$ in (2.15) and (2.20) and grouping like terms, we obtain

$$
\begin{aligned}
& 4(n-1) \sum_{|i|=2} a_{i, \pi(i)}+(4 n-2) \sum_{|i| \geq 3} a_{i, \pi(i)}-2 \sum_{|i| \geq 3} a_{\pi(i), i} \\
& +2\left(n-c_{1}(\pi)+2\right) \sum_{|i|=1} a_{i, i}-2\left(c_{1}(\pi)-2\right) \sum_{|i| \geq 2} a_{i, i} \\
& +4 \sum_{|i| \wedge|j|=1, j \neq i} a_{i, j}-2 \sum_{|i|=1,|j| \geq 2} a_{i, j}-2 \sum_{|i| \geq 2,|j|=1} a_{i, j} .
\end{aligned}
$$

The assumption $a_{i, i}=0$ causes the contribution from (2.22) to vanish, the assumption that there are no 1-cycles causes the contribution from (2.23) to vanish, and the assumption that $a_{i, j}$ is symmetric causes the combination of the second and third terms in (2.21) to yield $\mathrm{E}\left(Y^{\prime}-Y^{\prime \prime} \mid \pi^{\prime}\right)=(4 / n) \sum_{i=1}^{n} a_{i, \pi^{\prime}(i)}=(4 / n) Y^{\prime}$. Hence, the linearity condition $(2.1)$ is satisfied.

Since $\pi^{\prime \prime}=\tau_{I J} \pi \tau_{I J}$, the terms that multiply the indicator functions in the difference $Y^{\prime}-Y^{\prime \prime}$ in (2.13) depend only on values in a subset of $\left\{\pi^{-1}(I), I, \pi(I), \pi^{-1}(J), J, \pi(J)\right\}$ determined by the event indicated; for example, on $B_{I, J}$ the difference depends only on $\left\{\pi^{-1}(I), I, J, \pi(J)\right\}$. For each event, we tabulate such values in a vector $i$. Likewise, with $\pi^{\dagger}$ and $\pi^{\dagger}$ constructed according to $\pi^{\dagger}=\tau_{I^{\dagger} J^{\dagger}} \pi^{\dagger} \tau_{I^{\dagger} J^{\dagger}}$, the difference $Y^{\dagger}-Y^{\ddagger}$ depends only on a subset of $\left\{P^{\dagger}, I^{\dagger}, K^{\dagger}, Q^{\dagger}, J^{\dagger}, L^{\dagger}\right\}$ (the corresponding values in the $\pi^{\dagger}$ cycle), which we will tabulate in a vector $\boldsymbol{i}^{\dagger}$. Since $Y^{\prime}-Y^{\prime \prime}$ in (2.13) is a sum of terms multiplied by indicator functions of disjoint events, $\left(Y^{\prime}-Y^{\prime \prime}\right)^{2}$ is a sum of those terms squared, multiplied by the same indicator functions. Hence, to generate $\left(\pi^{\dagger}, \pi^{\ddagger}\right)$ such that $\left(Y^{\dagger}, Y^{\ddagger}\right)$ has a distribution proportional to $\left(y^{\prime}-y^{\prime \prime}\right)^{2} \mathrm{~d} F\left(y^{\prime}, y^{\prime \prime}\right)$, on each event we generate the elements of $i^{\dagger}$ with square-weighted probability appropriate to the set indicated. Once the values in $i^{\dagger}$ are chosen, in order for $\pi^{\dagger}$ to have the conditional distribution of $\pi$ (given these values), the remaining values of $\pi^{\dagger}$ are obtained by interchanging $\boldsymbol{i}$ and $\boldsymbol{i}^{\dagger}$ in the cycle structure of $\pi$. That is, in each case we specify $\pi^{\dagger}$ in terms of $\pi$ by

$$
\pi^{\dagger}=\tau_{i, i^{\dagger}} \pi \tau_{i, i^{\dagger}}, \quad \text { where } \quad \tau_{i, i^{\dagger}}=\prod_{k=1}^{\kappa} \tau_{i_{k}, i_{k}^{\dagger}}
$$

and $\boldsymbol{i}=\left(i_{1}, \ldots, i_{\kappa}\right)$ and $\boldsymbol{i}^{\dagger}=\left(i_{1}^{\dagger}, \ldots, i_{\kappa}^{\dagger}\right)$ are vectors of disjoint indices of some length $\kappa$.

For $\rho \in \ell_{\kappa}$ and $\boldsymbol{l}=\left(l_{1}, \ldots, l_{\kappa}\right)$ any $\kappa$-dimensional vector of indices, let $\rho(\boldsymbol{l})=\left\{\rho\left(l_{k}\right), k=\right.$ $1, \ldots, \kappa\}$ and let $\iota$ be the identity permutation. Since the values of $\tau_{i i} \dagger \pi \tau_{i i} \dagger$ may differ from those of $\pi$ only at $i, i^{\dagger}, \pi^{-1}(i)$, and $\pi^{-1}\left(i^{\dagger}\right),(2.5)$ will hold for the variables given by (2.8), with

$$
\tau=\iota(\boldsymbol{i}) \cup \iota\left(\boldsymbol{i}^{\dagger}\right) \cup \pi^{-1}(\boldsymbol{i}) \cup \pi^{-1}\left(\boldsymbol{i}^{\dagger}\right)
$$

The construction in each case proceeds as follows. Since 1-cycles are excluded, $A_{I, J}$ and $A_{J, I}$ are null. On $B_{I, J}$, where $I, J$, and $\pi(J)$ are three distinct, consecutive values of $\pi$, if $|I|=3$ then the symmetry of $a_{i, j}$ gives $Y^{\prime \prime}=Y^{\prime}$, an event on which the distribution of $\left(Y^{\dagger}, Y^{\ddagger}\right)$, proportional to $\left(Y^{\prime \prime}-Y^{\prime}\right)^{2}$, puts mass 0 . Otherwise, $|I| \geq 4$ and $Y^{\prime}-Y^{\prime \prime}$ depends only on 
$i=\left(\pi^{-1}(I), I, J, \pi(J)\right)$, and we choose $i^{\dagger}=\left(P^{\dagger}, I^{\dagger}, J^{\dagger}, L^{\dagger}\right)$, the corresponding values for $\pi^{\dagger}$, according to the distribution

$$
\left(P^{\dagger}, I^{\dagger}, J^{\dagger}, L^{\dagger}\right) \sim\left[\left(a_{p, i}+a_{j, l}\right)-\left(a_{p, j}+a_{i, l}\right)\right]^{2} \mathbf{1}(p, i, j, \text { and } l \text { are distinct }),
$$

noting that $a_{i, j}$ cancels with $a_{j, i}$ by symmetry. Now set $\pi^{\dagger}$ as specified in (2.24). In this case, $\tau$ has size at most thirteen. Reversing the roles of $I$ and $J$ gives the construction on $B_{J, I}$.

Next consider $F$, where $I, \pi(I), J$, and $\pi(J)$ are distinct. For $|I|=|J|=2$, take

$$
\left(I^{\dagger}, K^{\dagger}, J^{\dagger}, L^{\dagger}\right) \sim\left[\left(a_{i, k}+a_{j, l}\right)-\left(a_{i, l}+a_{j, k}\right)\right]^{2} \mathbf{1}(i, k, j, \text { and } l \text { are distinct })
$$

and set $\pi^{\dagger}$ as specified in (2.24) with $\boldsymbol{i}=(I, \pi(I), J, \pi(J))$ and $\boldsymbol{i}^{\dagger}=\left(I^{\dagger}, K^{\dagger}, J^{\dagger}, L^{\dagger}\right)$, and with the size of $\mathcal{I}$ at most twelve. For $|I| \geq 3$ and $|J|=2$, take

$$
\begin{aligned}
\left(P^{\dagger}, I^{\dagger}, K^{\dagger}, J^{\dagger}, L^{\dagger}\right) \sim & {\left[\left(a_{p, i}+a_{i, k}+2 a_{j, l}\right)-\left(a_{p, j}+a_{j, k}+2 a_{i, l}\right)\right]^{2} } \\
& \times \mathbf{1}(p, i, k, j, \text { and } l \text { are distinct })
\end{aligned}
$$

and set $\pi^{\dagger}$ as specified in (2.24) with $\boldsymbol{i}=\left(\pi^{-1}(I), I, \pi(I), J, \pi(J)\right)$ and $\boldsymbol{i}^{\dagger}=\left(P^{\dagger}, I^{\dagger}, K^{\dagger}, J^{\dagger}\right.$, $\left.L^{\dagger}\right)$, and with the size of $\mathcal{I}$ at most sixteen. Reversing the roles of $I$ and $J$ gives the case in which $|J|=2$ but $|I| \geq 3$. For $|I| \geq 3$ and $|J| \geq 3$, take

$$
\begin{aligned}
\left(P^{\dagger}, I^{\dagger}, K^{\dagger}, Q^{\dagger}, J^{\dagger}, L^{\dagger}\right) \sim & {\left[\left(a_{p, i}+a_{i, k}+a_{q, j}+a_{j, l}\right)-\left(a_{p, j}+a_{j, k}+a_{q, i}+a_{i, l}\right)\right]^{2} } \\
& \times \mathbf{1}(p, i, k, q, j, \text { and } l \text { are distinct }),
\end{aligned}
$$

and set $\pi^{\dagger}$ as specified in (2.24) with $i=\left(\pi^{-1}(I), I, \pi(I), \pi^{-1}(J), J, \pi(J)\right)$ and $\boldsymbol{i}^{\dagger}=$ $\left(P^{\dagger}, I^{\dagger}, K^{\dagger}, Q^{\dagger}, J^{\dagger}, L^{\dagger}\right)$. In this case, the size of $\mathcal{I}$ is at most twenty and, by (2.6), $\left|Y^{*}-Y\right| \leq$ $40 C$ in all cases.

\section{Size biasing: permutations and patterns}

In this section, we derive corollaries to Theorem 1.2 to obtain Berry-Esseen bounds for the number of occurrences of fixed, relatively ordered subsequences, such as rising sequences, in a random permutation, and of color patterns, local maxima, and subgraphs in finite graphs.

Following [17], given a finite collection $X=\left\{X_{\alpha}, \alpha \in \mathcal{A}\right\}$ of nonnegative random variables with index set $\mathcal{A}$, for $\alpha \in \mathcal{A}$ we say that the collection $\boldsymbol{X}^{\alpha}=\left\{X_{\beta}^{\alpha}, \beta \in \mathcal{A}\right\}$ has the $\boldsymbol{X}$-size-biased distribution in direction $\alpha$ if

$$
\mathrm{E} X_{\alpha} f(\boldsymbol{X})=\mathrm{E} X_{\alpha} \mathrm{E} f\left(\boldsymbol{X}^{\alpha}\right)
$$

for all functions $f$ on $\boldsymbol{X}$ for which these expectations exist. For the given $\boldsymbol{X}$, the collection $\boldsymbol{X}^{\alpha}$ exists for any $\alpha \in \mathcal{A}$ and has distribution $\mathrm{d} P^{\alpha}(\boldsymbol{x})=x_{\alpha} \mathrm{d} P(\boldsymbol{x}) / \mathrm{E} X_{\alpha}$, where $\mathrm{d} P(\boldsymbol{x})$ is the distribution of $\boldsymbol{X}$. Specializing (3.1) to the coordinate function $f(\boldsymbol{X})=g\left(X_{\alpha}\right)$, we see that $X_{\alpha}^{\alpha}$ has the $X_{\alpha}$-size-biased distribution $X_{\alpha}^{\mathrm{s}}$, defined in (1.4).

Corollary 3.1. Let $\boldsymbol{X}=\left\{X_{\alpha}, \alpha \in \mathcal{A}\right\}$ be a finite collection of random variables with values in $[0, M]$, and let $Y=\sum_{\alpha \in \mathcal{A}} X_{\alpha}$. Assume, for each $\alpha \in \mathcal{A}$, that there exists a dependency neighborhood $\mathcal{B}_{\alpha} \subset \mathcal{A}$ such that

$$
X_{\alpha} \text { and }\left\{X_{\beta}, \beta \notin \mathcal{B}_{\alpha}\right\} \text { are independent. }
$$


Furthermore, let $p_{\alpha}=\mathrm{E} X_{\alpha} / \sum_{\beta \in \mathcal{A}} \mathrm{E} X_{\beta}$ and $\max _{\alpha}\left|\mathscr{B}_{\alpha}\right|=b$. For each $\alpha \in \mathcal{A}$, let $\left(\boldsymbol{X}, \boldsymbol{X}^{\alpha}\right)$ be a coupling of $\boldsymbol{X}$ to an $\boldsymbol{X}^{\alpha}$ with the $\boldsymbol{X}$-size-biased distribution in direction $\alpha$, and let $\mathscr{D} \subset \mathcal{A} \times \mathcal{A}$ and $\mathcal{F} \supset \sigma\{Y\}$ be such that if $\left(\alpha_{1}, \alpha_{2}\right) \notin \mathscr{D}$, then

$$
\operatorname{cov}\left(\mathrm{E}\left(X_{\beta_{1}}^{\alpha_{1}}-X_{\beta_{1}} \mid \mathcal{F}\right), \mathrm{E}\left(X_{\beta_{2}}^{\alpha_{2}}-X_{\beta_{2}} \mid \mathcal{F}\right)\right)=0 \text { for all }\left(\beta_{1}, \beta_{2}\right) \in \mathcal{B}_{\alpha_{1}} \times \mathcal{B}_{\alpha_{2}} .
$$

Then Theorem 1.2 may be applied, with

$$
B=b M \quad \text { and } \quad \Delta \leq M \sqrt{\sum_{\left(\alpha_{1}, \alpha_{2}\right) \in \mathcal{D}} p_{\alpha_{1}} p_{\alpha_{2}}\left|\mathcal{B}_{\alpha_{1}}\right|\left|\mathcal{B}_{\alpha_{2}}\right|} \leq\left(\max _{\alpha} p_{\alpha}\right) b M \sqrt{|\mathscr{D}|} .
$$

Proof. Assuming, without loss of generality, that $\mathrm{E} X_{\alpha}>0$ for each $\alpha \in \mathcal{A}$, the factorization

$$
P^{\alpha}(\boldsymbol{X} \in \mathrm{d} \boldsymbol{x})=\left(\frac{x_{\alpha} P\left(X_{\alpha} \in \mathrm{d} x_{\alpha}\right)}{\mathrm{E} X_{\alpha}}\right) P\left(\boldsymbol{X} \in \mathrm{d} \boldsymbol{x} \mid X_{\alpha}=x_{\alpha}\right)
$$

shows that we can construct $\boldsymbol{X}^{\alpha}$ by first choosing $X_{\alpha}^{\alpha}$ from the $X_{\alpha}$-size-biased distribution, and then choosing the remaining variables from the conditional distribution of $\boldsymbol{X}$, given the chosen value of $X_{\alpha}^{\alpha}$. Note that $X_{\beta}^{\alpha} \in[0, M]$ for all $\alpha$ and $\beta$ and, by (3.2), that we may take $X_{\beta}^{\alpha}=X_{\beta}$ for $\beta \notin \mathscr{B}_{\alpha}$. By Lemma 2.1 of [17], $Y^{\mathrm{s}}=\sum_{\beta \in \mathcal{A}} X_{\beta}^{I}$ has the $Y$-size-biased distribution, where the random index $I$ has distribution $P(I=\alpha)=p_{\alpha}$, and is independent of both $\left(X, X^{\alpha}\right)$ and $\mathcal{F}$. Hence,

$$
Y^{\mathrm{s}}-Y=\sum_{\beta \in B_{I}}\left(X_{\beta}^{I}-X_{\beta}\right) \quad \text { and, therefore, } \quad\left|Y^{\mathrm{s}}-Y\right| \leq b M .
$$

Since $\sigma\{Y\} \subset \mathcal{F}$, we have $\Delta^{2}=\operatorname{var}\left(\mathrm{E}\left(Y^{\mathrm{s}}-Y \mid Y\right)\right) \leq \operatorname{var}\left(\mathrm{E}\left(Y^{\mathrm{s}}-Y \mid \mathcal{F}\right)\right)$. Taking the conditional expectation with respect to $\mathcal{F}$ in (3.5) yields

$$
\mathrm{E}\left(Y^{\mathrm{s}}-Y \mid \mathcal{F}\right)=\sum_{\alpha \in \mathcal{A}} p_{\alpha} \sum_{\beta \in \mathcal{B}_{\alpha}} \mathrm{E}\left(X_{\beta}^{\alpha}-X_{\beta} \mid \mathcal{F}\right)
$$

and, therefore,

$$
\operatorname{var}\left(\mathrm{E}\left(Y^{\mathrm{s}}-Y \mid \mathcal{F}\right)\right)=\mathrm{E} \sum_{\substack{\left(\alpha_{1}, \alpha_{2}\right) \in \mathcal{A} \times \mathcal{A} \\\left(\beta_{1}, \beta_{2}\right) \in \mathcal{B}_{\alpha_{1}} \times \mathcal{B}_{\alpha_{2}}}} p_{\alpha_{1}} p_{\alpha_{2}} \operatorname{cov}\left(\mathrm{E}\left(X_{\beta_{1}}^{\alpha_{1}}-X_{\beta_{1}} \mid \mathcal{F}\right), \mathrm{E}\left(X_{\beta_{2}}^{\alpha_{2}}-X_{\beta_{2}} \mid \mathcal{F}\right)\right)
$$

Using (3.3), we may replace the sum over $\left(\alpha_{1}, \alpha_{2}\right) \in \mathcal{A} \times \mathcal{A}$ by the sum over $\left(\alpha_{1}, \alpha_{2}\right) \in \mathscr{D}$, and subsequent application of the Cauchy-Schwarz inequality yields the bound (3.4) for $\Delta$.

If, in some asymptotic regime, the $X_{\alpha}$ are comparable in expectation in such a way that $p_{\alpha} \sim|\mathcal{A}|^{-1}$, if $\mu$ and $\sigma^{2}$ grow like $|\mathcal{A}|$, if $b$ remains bounded, and if $|\mathscr{D}|$ is of order $|\mathcal{A}|$, then, in Theorem 1.2, $A$ and $\Delta$ and, therefore, $\delta$ are of order $1 / \sigma$.

Corollary 3.2. Let $g$ be an index set, let $\left\{C_{g}, g \in \mathcal{G}\right\}$ be a collection of independent random elements taking values in an arbitrary set $\mathcal{C}$, let $\left\{g_{\alpha}, \alpha \in \mathcal{A}\right\}$ be a finite collection of subsets of $g$, and, for $\alpha \in \mathcal{A}$, let

$$
X_{\alpha} \equiv X_{\alpha}\left(C_{g}, g \in g_{\alpha}\right)
$$


be a function of the variables $\left\{C_{g}, g \in g_{\alpha}\right\}$ taking values in $[0, M]$. Then Theorem 1.2 may be applied to $Y=\sum_{\alpha} X_{\alpha}$ with $B$ and $\Delta$ as in (3.4), taking $p_{\alpha}=\mathrm{E} X_{\alpha} / \sum_{\beta} \mathrm{E} X_{\beta}$,

$$
\mathcal{B}_{\alpha}=\left\{\beta \in \mathcal{A}: g_{\beta} \cap g_{\alpha} \neq \varnothing\right\}, \quad \alpha \in \mathcal{A},
$$

and any $\mathcal{D}$ for which

$\mathscr{D} \supset\left\{\left(\alpha_{1}, \alpha_{2}\right)\right.$ : there exist $\beta_{1} \in \mathscr{B}_{\alpha_{1}}$ and $\beta_{2} \in \mathscr{B}_{\alpha_{2}}$ with $\left.g_{\beta_{1}} \cap g_{\beta_{2}} \neq \varnothing\right\}$.

Proof. Since $X_{\alpha}$ and $X_{\beta}$ are functions of disjoint sets of independent variables when $g_{\alpha} \cap$ $g_{\beta}=\varnothing$, (3.2) holds with the dependency neighborhoods given by (3.6). Now, for each $\alpha \in \mathcal{A}$, consider the following $\left(\boldsymbol{X}, \boldsymbol{X}^{\alpha}\right)$ coupling. Let $\left\{C_{g}^{(\alpha)}, g \in g_{\alpha}\right\}$ be independent of $\left\{C_{g}, g \in g\right\}$ and have distribution

$$
\mathrm{d} P^{(\alpha)}\left(c_{g}, g \in g_{\alpha}\right)=\frac{X_{\alpha}\left(c_{g}, g \in g_{\alpha}\right)}{\mathrm{E} X_{\alpha}\left(c_{g}, g \in g_{\alpha}\right)} \mathrm{d} P\left(c_{g}, g \in g_{\alpha}\right) .
$$

Then, by direct verification of (3.1), the collection

$$
X_{\beta}^{\alpha}=X_{\beta}\left(C_{g}, g \in g_{\beta} \cap g_{\alpha}^{c} ; C_{g}^{(\alpha)}, g \in g_{\beta} \cap g_{\alpha}\right), \quad \beta \in \mathcal{A}
$$

has the $X^{\alpha}$ distribution. Taking $\mathcal{F}=\left\{C_{g}, g \in g\right\}$, we have $\mathrm{E}\left(X_{\beta}^{\alpha} \mid \mathcal{F}\right)=\mathrm{E}\left(X_{\beta}^{\alpha} \mid C_{g}, g \in\right.$ $\left.g_{\beta}\right)$ and, since $\mathrm{E}\left(X_{\beta} \mid \mathcal{F}\right)=X_{\beta}$, the conditional expectation $\mathrm{E}\left(X_{\beta}^{\alpha}-X_{\beta} \mid \mathcal{F}\right)$ is a function of $\left\{C_{g}, g \in \mathscr{G}_{\beta}\right\}$ only. In particular, if $\left(\alpha_{1}, \alpha_{2}\right) \notin \mathscr{D}$ then, for all $\beta_{1} \in \mathscr{B}_{\alpha_{1}}$ and $\beta_{2} \in \mathscr{B}_{\alpha_{2}}$, we have $g_{\beta_{1}} \cap g_{\beta_{2}}=\varnothing$ and, consequently, $\mathrm{E}\left(X_{\beta_{1}}^{\alpha_{1}}-X_{\beta_{1}} \mid \mathcal{F}\right)$ and $\mathrm{E}\left(X_{\beta_{2}}^{\alpha_{2}}-X_{\beta_{2}} \mid \mathcal{F}\right)$ are independent, yielding (3.3), and all conditions of Corollary 3.1 hold.

With the exception of Example 3.2, in the remainder of this section we consider graphs $g=$

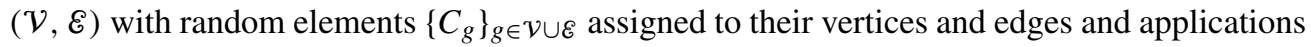
of Corollary 3.2 to the sum $Y=\sum_{\alpha \in \mathcal{A}} X_{\alpha}$ of bounded functions $X_{\alpha}=X_{\alpha}\left(C_{g}, g \in \mathcal{V}_{\alpha} \cup \varepsilon_{\alpha}\right)$, where $g_{\alpha}=\left(\mathcal{V}_{\alpha}, \mathcal{E}_{\alpha}\right), \alpha \in \mathcal{A}$, is a given finite family of subgraphs of $g_{\text {; }}$ we abuse notation slightly in that a graph $g$ is replaced by $\mathcal{V} \cup \mathcal{E}$ when used as an index set for the underlying variables $C_{g}$. When the elements of $\left\{C_{g}\right\}_{g \in g}$ are independent, Corollary 3.2 applies and, in (3.6) and (3.7), the intersection of the two graphs $\left(\mathcal{V}_{1}, \mathcal{E}_{1}\right)$ and $\left(\mathcal{V}_{2}, \mathcal{E}_{2}\right)$ is the graph $\left(\mathcal{V}_{1} \cap \mathcal{V}_{2}, \mathcal{E}_{1} \cap \mathcal{E}_{2}\right)$.

Furthermore, if $\mathcal{A} \subset \mathcal{V}$ and there is a distance $d(\alpha, \beta)$ defined on $\mathcal{A}$, then by letting

$$
\rho=\inf \left\{\varrho: \mathcal{V}_{\alpha} \cap \mathcal{V}_{\beta}=\varnothing \text { for all } \alpha, \beta \in \mathcal{A} \text { with } d(\alpha, \beta)>\varrho\right\},
$$

we may use

$$
\mathcal{B}_{\alpha}=\{\beta: d(\alpha, \beta) \leq \rho\} \quad \text { and } \quad \mathcal{D}=\left\{\left(\alpha_{1}, \alpha_{2}\right): d\left(\alpha_{1}, \alpha_{2}\right) \leq 3 \rho\right\}
$$

in (3.6) and (3.7), respectively, since rearranging $d\left(\alpha_{1}, \alpha_{2}\right) \leq d\left(\alpha_{1}, \beta_{1}\right)+d\left(\beta_{1}, \beta_{2}\right)+d\left(\beta_{2}, \alpha_{2}\right)$ gives

$$
d\left(\beta_{1}, \beta_{2}\right) \geq d\left(\alpha_{1}, \alpha_{2}\right)-\left(d\left(\alpha_{1}, \beta_{1}\right)+d\left(\alpha_{2}, \beta_{2}\right)\right) \geq d\left(\alpha_{1}, \alpha_{2}\right)-2 \rho>\rho
$$

for $\left(\alpha_{1}, \alpha_{2}\right) \notin \mathscr{D}$ and $\left(\beta_{1}, \beta_{2}\right) \in \mathscr{B}_{\alpha_{1}} \times \mathscr{B}_{\alpha_{2}}$.

For $v \in \mathcal{V}$ and $r \geq 0$, let $g_{v, r}$ be the restriction of $g_{\text {to }}$ to the vertices at most a distance $r$ from $v$; that is, $g_{v, r}$ has vertex set $\mathcal{V}_{v, r}=\{w \in \mathcal{V}: d(v, w) \leq r\}$ and edge set $\mathcal{E}_{v, r}=\{\{w, u\} \in$ $\left.\mathcal{E}: w, u \in \mathcal{V}_{v, r}\right\}$. We say that a graph $g$ is distance $r$-regular if $g_{v, r}$ is isomorphic to some 
graph $\left(\mathcal{V}_{r}, \mathcal{E}_{r}\right)$ for all $v$. For example, a graph of constant degree is distance 1-regular. This notion of distance $r$-regularity is related to, but not the same as, the notion of a distance-regular graph discussed in [6] and [9]. For a distance- $r$-regular graph, let

$$
V(r)=\left|\mathcal{V}_{r}\right| .
$$

Corollary 3.3, below, follows from Corollary 3.2 as a consequence of the remarks above, and by noting that the given assumptions imply that $|\mathscr{D}|=|\mathcal{A}| V(3 \rho)$ and that $\mathrm{E} X_{\alpha}$ is constant, yielding $p_{\alpha}=1 /|\mathcal{A}|$.

Corollary 3.3. Let $g$ be a graph with a finite family of isomorphic subgraphs $\left\{\mathcal{g}_{\alpha}, \alpha \in \mathcal{A}\right\}$, $\mathcal{A} \subset \mathcal{V}$, let $d(\cdot, \cdot)$ be a distance on $\mathcal{A}$, and define $\rho$ as in (3.8). For each $\alpha \in \mathcal{A}$, let $X_{\alpha}$ be given by

$$
X_{\alpha}=X\left(C_{g}, g \in g_{\alpha}\right)
$$

for a fixed function $X$ taking values in $[0, M]$, and let the elements of $\left\{C_{g}\right\}_{g \in g}$ be independent, with $\left\{C_{g}, g \in g_{\alpha}\right\}$ identically distributed. If $g$ is a distance-3 $\rho$-regular graph, then Theorem 1.2 may be applied to $Y=\sum_{\alpha \in \mathcal{A}} X_{\alpha}$ with $V(r)$ as given in (3.10) and

$$
B=V(\rho) M, \quad \Delta \leq M|\mathcal{A}|^{-1 / 2} V(\rho) \sqrt{V(3 \rho)} .
$$

Natural families of examples in $\mathbb{R}^{p}$ can be generated using the vertex set $\mathcal{V}=\{1, \ldots, n\}^{p}$, with componentwise addition modulo $n$, and $d(\alpha, \beta)$ given by, e.g. the $L^{1}$-distance $\|\alpha-\beta\|$.

Example 3.1. (Sliding $m$-window.) For $n \geq m \geq 1$, let $\mathcal{A}=\mathcal{V}=\{1, \ldots, n\}$, considered modulo $n$, let $\left\{C_{g}, g \in g\right\}$ be independent and identically distributed real-valued random variables, and, for each $\alpha \in \mathcal{A}$, let

$$
g_{\alpha}=\left(\mathcal{V}_{\alpha}, \mathcal{E}_{\alpha}\right), \quad \text { where } \mathcal{V}_{\alpha}=\{v \in \mathcal{V}: \alpha \leq v \leq \alpha+m-1\} \quad \text { and } \quad \varepsilon_{\alpha}=\varnothing .
$$

Then, for $X: \mathbb{R}^{m} \rightarrow[0,1]$, Corollary 3.3 may be applied to the sum $Y=\sum_{\alpha \in \mathcal{A}} X_{\alpha}$ of the $m$-dependent sequence $X_{\alpha}=X\left(C_{\alpha}, \ldots, C_{\alpha+m-1}\right)$, formed by applying the function $X$ to the variables in the ' $m$-window' $\mathcal{V}_{\alpha}$. In this example, taking $d(\alpha, \beta)=|\alpha-\beta|$ gives $\rho=m-1$ and $V(r)=2 r+1$. Hence, from (3.12), $B=(2 m-1)$ and $\Delta \leq n^{-1 / 2}(2 m-1)(6 m-5)^{1 / 2}$.

In Example 3.2, the underlying variables are not independent, and Corollaries 3.2 and 3.3 cannot be directly applied.

Example 3.2. (Relatively ordered subsequences of a random permutation.) For $n \geq m \geq 1$, let $\pi$ be a uniform random permutation of the integers $\mathcal{V}=\{1, \ldots, n\}$, taken modulo $n$. For a permutation $\tau$ on $\{1, \ldots, m\}$, let $g_{\alpha}$ and $\mathcal{V}_{\alpha}$ be as specified in (3.13), and let $X_{\alpha}$ be the indicator function requiring that the pattern $\tau$ appears in $\mathcal{V}_{\alpha}$; that is, that the values $\{\pi(v)\}_{v \in \mathcal{V}_{\alpha}}$ and $\{\tau(v)\}_{v \in \mathcal{V}_{1}}$ are in the same relative order. Equivalently, the pattern $\tau$ appears in $\mathcal{V}_{\alpha}$ if and only if $\pi\left(\tau^{-1}(v)+\alpha-1\right), v \in \mathcal{V}_{1}$, is an increasing sequence, and we write

$$
X_{\alpha}\left(\pi(v), v \in g_{\alpha}\right)=\mathbf{1}\left(\pi\left(\tau^{-1}(1)+\alpha-1\right)<\cdots<\pi\left(\tau^{-1}(m)+\alpha-1\right)\right) .
$$

With $\mathcal{A}=\mathcal{V}$, the sum $Y=\sum_{\alpha \in \mathcal{A}} X_{\alpha}$ counts the number of $m$-element-long segments of $\pi$ that have the same relative order as $\tau$.

For $\alpha \in \mathcal{A}$, we generate $\boldsymbol{X}^{\alpha}=\left\{X_{\beta}^{\alpha}, \beta \in \mathcal{A}\right\}$ by reordering the values of $\pi(\gamma), \gamma \in \mathcal{V}_{\alpha}$, to be in the same relative order as $\tau$, and let $X_{\beta}^{\alpha}$ be the indicator function requiring $\tau$ to appear at 
position $\beta$ in the reordered permutation. Letting $\mathcal{F}=\sigma\{\pi\}$, we have $\mathrm{E}\left(X_{\beta}^{\alpha} \mid \mathcal{F}\right)$ and the $X_{\beta}$ depend only on the relative order of $\{\pi(\gamma),-(m-1) \leq \gamma-\beta \leq 2(m-1)\}$. Since the relative orders of the non-overlapping segments of the values of $\pi$ are independent, (3.2) and (3.3) hold when $\mathcal{B}_{\alpha}$ and $\mathscr{D}$ are as in (3.9), for $d(\alpha, \beta)=|\alpha-\beta|$ and $\rho=m-1$; hence, Theorem 1.2 may be applied with the same value for $B$ and bound on $\Delta$ as in Example 3.1.

When $\tau=\iota_{m}$, the identity permutation of length $m$, we say that $\pi$ has a rising sequence of length $m$ at position $\alpha$ if $X_{\alpha}=1$. Rising sequences were studied in [4] in connection with card tricks and card shuffling. Owing to the regular-self-overlap property of rising sequences, namely that a nonempty intersection of two rising sequences is again a rising sequence, some improvement on the constant in the bound can be obtained by a more careful consideration of the conditional variance.

Example 3.3. (Coloring patterns and subgraph occurrences in a finite graph g.) For illustration, take $\mathcal{V}=\mathcal{A}=\{1, \ldots, n\}^{p}$, considered modulo $n$, let $d(\alpha, \beta)=\|\alpha-\beta\|$ with $\|\cdot\|$ the supremum norm, let $\mathcal{E}=\{\{w, v\}: d(w, v)=1\}$, and, for each $\alpha \in \mathcal{A}$, let $g_{\alpha}=\left(\mathcal{V}_{\alpha}, \mathcal{E}_{\alpha}\right)$, where

$$
\mathcal{V}_{\alpha}=\left\{\alpha+\left(e_{1}, \ldots, e_{p}\right): e_{i} \in\{0,1\}\right\} \quad \text { and } \quad \mathcal{E}_{\alpha}=\left\{\{v, w\}: v, w \in \mathcal{V}_{\alpha}, d(w, v)=1\right\} .
$$

Let $\mathcal{C}$ be a set (of, e.g. colors) from which is formed a given pattern $\left\{c_{g}, g \in g_{0}\right\}$, let $\left\{C_{g}, g \in g_{f}\right\}$ be independent variables in $\mathcal{C}$ with $\left\{C_{g}, g \in g_{\alpha}\right\}_{\alpha \in \mathcal{A}}$ identically distributed, and let

$$
X\left(C_{g}, g \in g_{0}\right)=\prod_{g \in g_{0}} \mathbf{1}\left(C_{g}=c_{g}\right),
$$

with $X_{\alpha}$ given by (3.11). Then, $Y=\sum_{\alpha \in \mathcal{A}} X_{\alpha}$ counts the number of times the pattern appears in the subgraphs $\mathcal{G}_{\alpha}$. Corollary 3.3 may be applied with $M=1, \rho=1$ (by (3.8)), $V(r)=(2 r+1)^{p}$, and (by (3.12)) $B=3^{p}$ and $\Delta \leq(63 / n)^{p / 2}$.

Such multidimensional pattern occurrences are a generalization of the well-studied case in which one-dimensional sequences are scanned for pattern occurrences; see, for instance, [13] and [23] for scan and window statistics, see [21] for applications of the normal approximation in this context to molecular sequence data, and see also [11] and [12], where higher-dimensional extensions are considered.

Subgraph occurrences can be handled as a special case. For example, with $(\mathcal{V}, \mathcal{E})$ the graph defined above, let $G$ be the random subgraph with vertex set $\mathcal{V}$ and random edge set $\left\{e \in \mathcal{E}: C_{e}=1\right\}$ where $\left\{C_{e}\right\}_{e \in \mathcal{E}}$ are independent and identically distributed Bernoulli variables. Then, taking the product in (3.14) over edges $e \in \mathcal{E}_{0}$, say, and setting $c_{e}=1$, the sum $Y=\sum_{\alpha \in \mathcal{A}} X_{\alpha}$ counts the number of times that copies of $\varepsilon_{0}$ appear in the random graph $G$; the same bounds hold as above.

The authors of [3] studied the related problem of counting the number of small cliques that occur in the random binomial graph, a case in which the dependence is not local; the technique applied is the Chen-Stein method.

Example 3.4. (Local extremes.) Let $g_{\alpha}, \alpha \in \mathcal{A}$, be a collection of subgraphs of $g_{\text {s }}$ isomorphic to $g_{0}$, let $v \in \mathcal{V}_{0}$ be a distinguished vertex, let $\left\{C_{g}, g \in \mathcal{V}\right\}$ be a collection of independent and identically distributed random variables, and let $X_{\alpha}$ be defined by (3.11), with

$$
X\left(C_{\beta}, \beta \in \mathcal{V}_{0}\right)=\mathbf{1}\left(C_{v} \geq C_{\beta}, \beta \in \mathcal{V}_{0}\right) .
$$

Then, the sum $Y=\sum_{\alpha \in \mathcal{A}} X_{\alpha}$ counts the number of times that the distinguished vertex in $g_{\alpha}$, which corresponds under the isomorphism to the distinguished vertex $v \in \mathcal{V}_{0}$, is a local 
maximum. Corollary 3.3 holds with $M=1$, the other quantities determining the bound being dependent on the structure of $g$.

For example, consider the hypercube with $\mathcal{V}=\{0,1\}^{p}$ and $\mathcal{E}=\{\{v, w\}:\|v-w\|=1\}$, where $\|\cdot\|$ is the Hamming distance (see [2] and [1]). Take $v=\mathbf{0}, \mathcal{A}=\mathcal{V}$, and, for each $\alpha \in \mathcal{A}$, let $\mathcal{V}_{\alpha}=\{\beta:\|\beta-\alpha\| \leq 1\}$ and $\varepsilon_{\alpha}=\left\{\{v, w\}: v, w \in \mathcal{V}_{\alpha},\|v-w\|=1\right\}$. Corollary 3.3 applies with $\rho=2$ (by (3.8)), $V(r)=\sum_{j=0}^{r}\left(\begin{array}{l}p \\ j\end{array}\right)$, and (by (3.12))

$$
B=1+p+\left(\begin{array}{l}
p \\
2
\end{array}\right) \quad \text { and } \quad \Delta \leq 2^{-p / 2} \sum_{j=0}^{2}\left(\begin{array}{l}
p \\
j
\end{array}\right) \sqrt{\sum_{j=0}^{6}\left(\begin{array}{l}
p \\
j
\end{array}\right)}
$$

\section{Proofs of Theorems 1.1 and 1.2}

In this section, $\mathscr{H}$ denotes a class of measurable functions satisfying properties (i), (ii), and (iii) (as described in Section 1) and $h$ denotes an element of $\mathcal{H}$. Recall that $\delta$ is given by (1.6), let $\phi(t)$ denote the standard normal density, and, for $t \in(0,1)$, define

$$
h_{t}(x)=\int h(x+t y) \phi(y) \mathrm{d} y \quad \text { and } \quad \delta_{t}=\sup \left\{\left|\mathrm{E} h_{t}(W)-N h_{t}\right|, h \in \mathscr{H}\right\} .
$$

Lemma 4.1. For a random variable $W$ on $\mathbb{R}$, we have

$$
\delta \leq 2.8 \delta_{t}+4.7 \text { at } \text { for all } t \in(0,1)
$$

where $a$ is as in (1.8). Furthermore, for all $A>0$ and $\tilde{h}_{\varepsilon}$ in as in (1.7),

$$
\mathrm{E}\left(\int \tilde{h}_{A+t|y|}(W)\left|\phi^{\prime}(y)\right| \mathrm{d} y\right) \leq 2 \delta+a(A+t) .
$$

Proof. Inequality (4.2) is Lemma 4.1 of [25], following Lemma 2.11 of [19], which stems from [5]. As in [25], adding to and subtracting from the left-hand side of (4.3) gives

$$
\begin{aligned}
& \mathrm{E}\left(\int\left(\tilde{h}_{A+t|y|}(W)-\tilde{h}_{A+t|y|}(Z)\right)\left|\phi^{\prime}(y)\right| \mathrm{d} y+\int \tilde{h}_{A+t|y|}(Z)\left|\phi^{\prime}(y)\right| \mathrm{d} y\right) \\
& \quad \leq \int\left|\mathrm{E} \tilde{h}_{A+t|y|}(W)-\mathrm{E} \tilde{h}_{A+t|y|}(Z)\right|\left|\phi^{\prime}(y)\right| \mathrm{d} y+\int \mathrm{E} \tilde{h}_{A+t|y|}(Z)\left|\phi^{\prime}(y)\right| \mathrm{d} y \\
& \quad \leq\left(2 \delta+\int a(A+t|y|)\left|\phi^{\prime}(y)\right| \mathrm{d} y\right) \leq 2 \delta+a(A+t),
\end{aligned}
$$

where, for the first term inside the parentheses in (4.4), we have used the facts that $h_{A+t|y|}^{ \pm} \in \mathscr{H}$ and $\int\left|\phi^{\prime}(y)\right| \mathrm{d} y \leq 1$. For the second term in the parentheses, we have used (1.8) and the fact that $\int|y|\left|\phi^{\prime}(y)\right| \mathrm{d} y=1$.

In Sections 4.1 and 4.2, $h_{t}$ is given by (4.1) and $f$ is the bounded solution of the Stein equation (1.2) with $\mu=0, \sigma^{2}=1$, and test function $h_{t}$. With $\|\cdot\|$ the supremum norm, Lemma 3 of [28] gives

$$
\|f\| \leq \sqrt{2 \pi} \leq 2.6 \text { and }\left\|f^{\prime}\right\| \leq 4 .
$$




\subsection{Proof of Theorem 1.1 (zero biasing)}

Lemma 4.2. Let $Y$ be a mean-zero random variable with variance $\sigma^{2}$, and let $Y^{*}$ be defined on the same space as $Y$, with the $Y$-zero-biased distribution, satisfying $\left|Y^{*}-Y\right| / \sigma \leq A$ for some A. Then

$$
\delta_{t} \leq(6.6+a) A+2 A^{2}+\frac{1}{t}\left(2 \delta A+a A^{2}\right) \quad \text { for all } t \in(0,1) .
$$

Proof. Let $W=Y / \sigma$, whence $W^{*}=Y^{*} / \sigma$ and $\left|W^{*}-W\right| \leq A$. By differentiation in (1.2) and (4.1), respectively, we have

$$
f^{\prime \prime}(x)=f(x)+x f^{\prime}(x)+h_{t}^{\prime}(x), \quad \text { with } \quad h_{t}^{\prime}(x)=-\frac{1}{t} \int h(x+t y) \phi^{\prime}(y) \mathrm{d} y .
$$

By (1.5) and (4.6), with $N h_{t}=\mathrm{E} h_{t}(Z)$ for a standard normal variable $Z$, we also have

$$
\begin{aligned}
\left|\mathrm{E} h_{t}(W)-N h_{t}\right| & =\left|\mathrm{E}\left(f^{\prime}\left(W^{*}\right)-f^{\prime}(W)\right)\right|=\left|\mathrm{E} \int_{W}^{W^{*}} f^{\prime \prime}(x) \mathrm{d} x\right| \\
& =\left|\mathrm{E} \int_{W}^{W^{*}}\left(f(x)+x f^{\prime}(x)+h_{t}^{\prime}(x)\right) \mathrm{d} x\right| .
\end{aligned}
$$

Let $V=W^{*}-W$. Applying the triangle inequality in (4.7) and using (4.5), for the first term we find that

$$
\left|\mathrm{E} \int_{W}^{W^{*}} f(x) \mathrm{d} x\right| \leq 2.6 \mathrm{E}|V| \leq 2.6 A
$$

and, for the second term, again using (4.5) and, now, $\mathrm{E}|W| \leq\left(\mathrm{E} W^{2}\right)^{1 / 2}=1$, we find that

$$
\begin{aligned}
\left|\mathrm{E} \int_{W}^{W^{*}} x f^{\prime}(x) \mathrm{d} x\right| & \leq 4 \mathrm{E}\left|\int_{W}^{W+V}\right| x|\mathrm{~d} x|=2 \mathrm{E}|(W+V)| W+V|-W| W|| \\
& \leq \mathrm{E}\left(4|W V|+2 V^{2}\right) \leq 4 A \mathrm{E}|W|+2 A^{2} \leq 4 A+2 A^{2} .
\end{aligned}
$$

For the final term in (4.7), with $U \sim \mathcal{U}[0,1]$ independent of $W$ and $V$, we write

$$
\left|\mathrm{E} \int_{W}^{W^{*}} h_{t}^{\prime}(x) \mathrm{d} x\right|=\left|\mathrm{E} V \int_{0}^{1} h_{t}^{\prime}(W+u V) \mathrm{d} u\right|=\left|\mathrm{E} V h_{t}^{\prime}(W+U V)\right| .
$$

Then, using (4.6), $\int \phi^{\prime}(y) \mathrm{d} y=0$, and Lemma 4.1, we have

$$
\begin{aligned}
\mid \mathrm{E} V & h_{t}^{\prime}(W+U V)\left|=\frac{1}{t}\right| \mathrm{E} V \int h(W+U V+t y) \phi^{\prime}(y) \mathrm{d} y \mid \\
& =\frac{1}{t}\left|\mathrm{E} V \int[h(W+U V+t y)-h(W+U V)] \phi^{\prime}(y) \mathrm{d} y\right| \\
& \leq \frac{1}{t} \mathrm{E}\left(|V| \int\left[h_{|V|+t|y|}^{+}(W)-h_{|V|+t|y|}^{-}(W)\right]\left|\phi^{\prime}(y)\right| \mathrm{d} y\right) \\
& \leq \frac{1}{t} A \mathrm{E}\left(\int \tilde{h}_{A+t|y|}(W)\left|\phi^{\prime}(y)\right| \mathrm{d} y\right) \\
& \leq \frac{1}{t} A(2 \delta+a(A+t))=\frac{1}{t}\left(2 \delta A+a A^{2}\right)+a A .
\end{aligned}
$$

By combining the bounds (4.8), (4.9), and (4.10), we complete the proof. 
Proof of Theorem 1.1. Letting $t=\alpha A$ in Lemma 4.2, we have

$$
\delta_{t} \leq(6.6+a) A+2 A^{2}+\frac{1}{\alpha A}\left(2 \delta A+a A^{2}\right)=\left(6.6+a+\frac{a}{\alpha}\right) A+2 A^{2}+\frac{2 \delta}{\alpha} .
$$

Substituting (4.11) into the bound for $\delta$ given by Lemma 4.1, we have

$$
\begin{aligned}
\delta & \leq 2.8\left(\left(6.6+a+\frac{a}{\alpha}\right) A+2 A^{2}+\frac{2 \delta}{\alpha}\right)+4.7 a \alpha A \\
& \leq 18.5 A+2.8 a A+2.8 \frac{a A}{\alpha}+5.6 A^{2}+5.6 \frac{\delta}{\alpha}+4.7 a \alpha A,
\end{aligned}
$$

meaning that

$$
\delta \leq A\left(\frac{18.5+5.6 A+2.8 a+2.8 a / \alpha+4.7 a \alpha}{1-5.6 / \alpha}\right)
$$

Setting $\alpha=2 \times 5.6$, for which $t<1$ since $A \leq \frac{1}{12}$, we obtain (1.9) and, hence, the theorem.

\subsection{Proof of Theorem 1.2 (size biasing)}

Lemma 4.3. Let $Y \geq 0$ be a random variable with mean $\mu$ and variance $\sigma^{2}$, and let $Y^{\mathrm{s}}$ be defined on the same space as $Y$, with the $Y$-size-biased distribution, satisfying $\left|Y^{\mathrm{s}}-Y\right| / \sigma \leq A$ for some A. Then, for all $t \in(0,1)$,

$$
\delta_{t} \leq \frac{\mu}{\sigma}\left(\frac{4 \Delta}{\sigma}+\left(3.3+\frac{1}{2} a\right) A^{2}+\frac{2}{3} A^{3}+\frac{1}{2 t}\left(2 \delta A^{2}+a A^{3}\right)\right),
$$

with $\Delta$ as in (1.13).

Proof. With $W=(Y-\mu) / \sigma$, let $W^{\mathrm{s}}=\left(Y^{\mathrm{s}}-\mu\right) / \sigma$ (which is a slight abuse of notation). Then, $\left|W^{\mathrm{s}}-W\right| \leq A$. Note that

$$
\mathrm{E} W f(W)=\frac{\mu}{\sigma}\left(f\left(W^{\mathrm{s}}\right)-f(W)\right)
$$

and, so, with $V=W^{\mathrm{s}}-W$, we have

$$
\begin{aligned}
\mathrm{E} h_{t}(W)-N h_{t}= & \mathrm{E}\left(f^{\prime}(W)-W f(W)\right) \\
= & \mathrm{E}\left(f^{\prime}(W)-\frac{\mu}{\sigma}\left(f\left(W^{\mathrm{s}}\right)-f(W)\right)\right) \\
= & \mathrm{E}\left(f^{\prime}(W)-\frac{\mu}{\sigma} \int_{W}^{W^{\mathrm{s}}} f^{\prime}(x) \mathrm{d} x\right) \\
= & \mathrm{E}\left(f^{\prime}(W)-\frac{\mu}{\sigma} V \int_{0}^{1} f^{\prime}(W+u V) \mathrm{d} u\right) \\
= & \mathrm{E}\left(f^{\prime}(W)-\frac{\mu}{\sigma} V f^{\prime}(W)\right) \\
& +\mathrm{E}\left(\frac{\mu}{\sigma} V f^{\prime}(W)-\frac{\mu}{\sigma} V \int_{0}^{1} f^{\prime}(W+u V) \mathrm{d} u\right) .
\end{aligned}
$$


Since

$$
\mathrm{E}\left(\frac{\mu V}{\sigma}\right)=\frac{\mu}{\sigma} \mathrm{E}\left(W^{\mathrm{s}}-W\right)=\frac{1}{\sigma^{2}} \mathrm{E}\left(\mu Y^{\mathrm{s}}-\mu Y\right)=1,
$$

for the first expectation in (4.15) we have

$$
\mathrm{E}\left(f^{\prime}(W) \mathrm{E}\left(1-\frac{\mu}{\sigma} V \mid W\right)\right) \leq 4 \frac{\mu}{\sigma} \sqrt{\operatorname{var}\left(\mathrm{E}\left(W^{\mathrm{s}}-W \mid W\right)\right)}=4 \frac{\mu}{\sigma^{2}} \Delta,
$$

using (4.5) and (1.13). Now, using (4.6), we write the second expectation in (4.15) as

$$
\begin{aligned}
\frac{\mu}{\sigma} V\left\{f^{\prime}(W)-\int_{0}^{1} f^{\prime}(W+u V) \mathrm{d} u\right\} & =\frac{\mu}{\sigma} V \int_{0}^{1}\left(f^{\prime}(W)-f^{\prime}(W+u V)\right) \mathrm{d} u \\
& =-\frac{\mu}{\sigma} V \int_{0}^{1} \int_{W}^{W+u V} f^{\prime \prime}(v) \mathrm{d} v \mathrm{~d} u \\
& =-\frac{\mu}{\sigma} V \int_{0}^{1} \int_{W}^{W+u V}\left(f(v)+v f^{\prime}(v)+h_{t}^{\prime}(v)\right) \mathrm{d} v \mathrm{~d} u
\end{aligned}
$$

We apply the triangle inequality and bound the three resulting terms separately. For the expectation arising from the first term on the right-hand side of (4.17), by (4.5) we have

$$
\left|\mathrm{E}\left(\frac{\mu}{\sigma} V \int_{0}^{1} \int_{W}^{W+u V} f(v) \mathrm{d} v \mathrm{~d} u\right)\right| \leq 2.6 \frac{\mu}{\sigma} \mathrm{E}\left(|V| \int_{0}^{1} u|V| \mathrm{d} u\right) \leq 1.3 \frac{\mu}{\sigma} A^{2}
$$

and, for the second term, arguing as in (4.9) we have

$$
\begin{aligned}
\left|\mathrm{E}\left(\frac{\mu}{\sigma} V \int_{0}^{1} \int_{W}^{W+u V} v f^{\prime}(v) \mathrm{d} v \mathrm{~d} u\right)\right| & \leq 2 \frac{\mu}{\sigma} \mathrm{E}|V| \int_{0}^{1}\left|\int_{W}^{W+u V} 2\right| v|\mathrm{~d} v| \mathrm{d} u \\
& \leq 2 \frac{\mu}{\sigma} \mathrm{E}|V| \int_{0}^{1}\left(2 u|W V|+u^{2} V^{2}\right) \mathrm{d} u \\
& \leq 2 \frac{\mu}{\sigma} A \int_{0}^{1}\left(2 A u \mathrm{E}|W|+u^{2} A^{2}\right) \mathrm{d} u \\
& \leq 2 \frac{\mu}{\sigma} A\left(A+\frac{A^{2}}{3}\right)
\end{aligned}
$$

For the last term in (4.17), the computation is more involved than, yet similar to, that for zero biasing. Beginning with the inner integral, we have

$$
\int_{W}^{W+u V} h_{t}^{\prime}(v) \mathrm{d} v=u V \int_{0}^{1} h_{t}^{\prime}(W+x u V) \mathrm{d} x
$$

and, using (4.6),

$$
\int \phi^{\prime}(y) \mathrm{d} y=0
$$


and Lemma 4.1, for the last term in (4.17) we have

$$
\begin{aligned}
\mid \frac{\mu}{\sigma} \mathrm{E} & \int_{0}^{1} \int_{0}^{1} u V^{2} h_{t}^{\prime}(W+x u V) \mathrm{d} x \mathrm{~d} u \mid \\
& =\frac{\mu}{\sigma t}\left|\mathrm{E} V^{2} \int_{0}^{1} \int_{0}^{1} \int u h(W+x u V+t y) \phi^{\prime}(y) \mathrm{d} y \mathrm{~d} x \mathrm{~d} u\right| \\
& =\frac{\mu}{\sigma t}\left|\mathrm{E} V^{2} \int_{0}^{1} \int_{0}^{1} \int u[h(W+x u V+t y)-h(W+x u V)] \phi^{\prime}(y) \mathrm{d} y \mathrm{~d} x \mathrm{~d} u\right| \\
& \leq \frac{\mu}{\sigma t} \mathrm{E}\left(V^{2} \iint_{0}^{1} u\left[h_{|V|+t|y|}^{+}(W)-h_{|V|+t|y|}^{-}(W)\right]\left|\phi^{\prime}(y)\right| \mathrm{d} u \mathrm{~d} y\right) \\
& =\frac{\mu}{2 \sigma t} \mathrm{E}\left(V^{2} \int\left[h_{|V|+t|y|}^{+}(W)-h_{|V|+t|y|}^{-}(W)\right]\left|\phi^{\prime}(y)\right| \mathrm{d} y\right) \\
& \leq \frac{\mu}{2 \sigma t} A^{2} \mathrm{E}\left(\int \tilde{h}_{A+t|y|}(W)\left|\phi^{\prime}(y)\right| \mathrm{d} y\right) \\
& \leq \frac{\mu}{2 \sigma t} A^{2}(2 \delta+a(A+t)) \\
& =\frac{\mu}{2 \sigma t}\left(2 \delta A^{2}+a A^{3}\right)+\frac{\mu}{2 \sigma} a A^{2} .
\end{aligned}
$$

By combining (4.16), (4.18), (4.19), and (4.20), we complete the proof.

Proof of Theorem 1.2. Applying Lemma 4.1 using the bound (4.13) on $\delta_{t}$, we have

$$
\delta \leq 2.8 \frac{\mu}{\sigma}\left(\frac{4 \Delta}{\sigma}+\left(3.3+\frac{1}{2} a\right) A^{2}+\frac{2}{3} A^{3}+\frac{1}{2 t}\left(2 \delta A^{2}+a A^{3}\right)\right)+4.7 a t,
$$

or

$$
\delta \leq \frac{2.8(\mu / \sigma)\left(4 \Delta / \sigma+\left(3.3+\frac{1}{2} a\right) A^{2}+\frac{2}{3} A^{3}+a A^{3} / 2 t\right)+4.7 a t}{1-2.8 \mu A^{2} / \sigma t} .
$$

Setting $t=2 \times 2.8 \mu A^{2} / \sigma$, such that $t<1$ since $A \leq(\sigma / 6 \mu)^{1 / 2},(1.12)$ now follows from

$$
\begin{aligned}
\delta & \leq 5.6 \frac{\mu}{\sigma}\left(\frac{4 \Delta}{\sigma}+\left(3.3+\frac{1}{2} a\right) A^{2}+\frac{2}{3} A^{3}+\frac{\sigma}{2 \cdot 5.6 \mu} a A\right)+2 \cdot 4.7 a\left(5.6 \frac{\mu A^{2}}{\sigma}\right) \\
& \leq \frac{a A}{2}+\frac{\mu}{\sigma}\left((19+56 a) A^{2}+4 A^{3}\right)+23 \frac{\mu \Delta}{\sigma^{2}} .
\end{aligned}
$$

There are compromises in the choice of smoothing parameter: if we take $\alpha=4 \times 5.6$ in (4.12) for $B \leq \frac{1}{48} \sigma$, and $t=4 \times 2.8 \mu A^{2} / \sigma$ in (4.21) for $B \leq \sigma^{3 / 2} /(12 \mu)^{1 / 2}$, bounds (1.9) and (1.12) become

$$
\delta \leq A(145 a+7.5 A+25)
$$

and

$$
\delta \leq \frac{a A}{6}+\frac{\mu}{\sigma}\left((13+73 a) A^{2}+2.5 A^{3}\right)+15 \frac{\mu \Delta}{\sigma^{2}},
$$

respectively. 


\section{Remarks}

The zero- and size-bias couplings both conform well to Stein's characterizing equation, and their use produces bounds on the distance of a random variable $Y$ to the normal in many instances. The couplings are adaptable to the situation; in particular, the size-bias coupling, previously used in [17] in the case of global dependence, is applied here to handle cases with local dependence.

The applications in Section 2 illustrate how bounds on the distance $\delta$ from $Y$ to the normal can be generated using only a zero-bias coupling and a bound on $\left|Y^{*}-Y\right|$; in particular, the bounds do not depend on the often difficult calculation of variances of conditional expectations of the form $\operatorname{var}(\mathrm{E}(\tilde{Y}-Y \mid Y))$, which appear in the exchangeable-pair and size-biased versions of Stein's method when coupling $Y$ to some $\tilde{Y}$. It is hoped that this feature of the zero-bias method will motivate a better understanding of the construction of couplings of $Y^{*}$ to $Y$ in greater generality than those that depend on the existence of the exchangeable pair of Proposition 2.1. In particular, the applications in Section 3 show an evidently wider scope of applicability of the size-bias coupling over the zero-bias one, as it is presently understood.

\section{Acknowledgements}

The author would like to thank Martin Raic and Qi-Man Shao for their insightful comments on an earlier version of this manuscript.

\section{References}

[1] Baldi, P. And Rinott, Y. (1989). Asymptotic normality of some graph-related statistics. J. Appl. Prob. 26, 171-175.

[2] Baldi, P., Rinott, Y. And Stein, C. (1989). A normal approximation for the number of local maxima of a random function on a graph. In Probability, Statistics, and Mathematics, Academic Press, Boston, MA, pp. 59-81.

[3] Barbour, A. D., Janson, S., Karoński, M. And RucińsKi, A. (1990). Small cliques in random graphs. Random Structures Algorithms 1, 403-434.

[4] Bayer, D. And Diaconis, P. (1992). Trailing the dovetail shuffle to its lair. Ann. Appl. Prob. 2, $294-313$.

[5] Bhattacharya, R. N. and Ranga Rao, R. (1986). Normal Approximation and Asymptotic Expansions. Krieger, Melbourne, FL.

[6] Biggs, N. (1993). Algebraic Graph Theory. Cambridge University Press.

[7] Bolthausen, E. (1984). An estimate of the remainder in a combinatorial central limit theorem. Z. Wahrscheinlichkeitsth. 66, 379-386.

[8] Bolthausen, E. and Götze, F. (1993). The rate of convergence for multivariate sampling statistics. Ann. Statist. 21, 1692-1710.

[9] Brouwer, A. E., Cohen, A. M. and Neumaier, A. (1989). Distance-Regular Graphs. Springer, Berlin.

[10] Chen, L. H. Y. AND Shao, Q.-M. (2004). Normal approximation under local dependence. Ann. Prob. 32, 19852028.

[11] Darling, R. W. R. and Waterman, M. S. (1986). Extreme value distribution for the largest cube in a random lattice. SIAM J. Appl. Math. 46, 118-132.

[12] Darling, R. W. R. and Waterman, M. S. (1985). Matching rectangles in $d$ dimensions: algorithms and laws of large numbers. Adv. Math. 55, 1-12.

[13] Glaz, J., Naus, J. And Wallenstein, S. (2001). Scan Statistics. Springer, New York.

[14] Goldstein, L. (2004). Normal approximation for hierarchical sequences. Ann. Appl. Prob. 14, 1950-1969.

[15] Goldstein, L. And Reinert, G. (1997). Stein's method and the zero bias transformation with application to simple random sampling. Ann. Appl. Prob. 7, 935-952.

[16] Goldstein, L. AND Reinert, G. (2005). Distributional transformations, orthogonal polynomials, and Stein characterizations. J. Theoret. Prob. 18, 185-208.

[17] Goldstein, L. And RinotT, Y. (1996). On multivariate normal approximations by Stein's method and size bias couplings. J. Appl. Prob. 33, 1-17.

[18] Goldstein, L. And RinotT, Y. (2004). A permutation test for matching and its asymptotic distribution. Metron 61, 375-388.

[19] Götze, F. (1991). On the rate of convergence in the multivariate CLT. Ann. Prob. 19, 724-739. 
[20] Ho, S. T. And CHEN, L. H. Y. (1978). An $L_{p}$ bound for the remainder in a combinatorial central limit theorem. Ann. Prob. 6, 231-249.

[21] Hunan, H. (2002). Error bounds on multivariate normal approximations for word count statistics. Adv. Appl. Prob. 34, 559-586.

[22] Kolchin, V. F. and Chistyakov, V. P. (1973). On a combinatorial limit theorem. Theory Prob. Appl. 18, 728-739.

[23] Naus, J. I. (1982). Approximations for distributions of scan statistics. J. Amer. Statist. Assoc. 77, $177-183$.

[24] Rinoti, Y. and Rotar, V. (1996). A multivariate CLT for local dependence with $n^{-1 / 2} \log n$ rate and applications to multivariate graph related statistics. J. Multivariate Anal. 56, 333-350.

[25] RinotT, Y. ANd RotaR, V. (1997). On coupling constructions and rates in the CLT for dependent summands with applications to the antivoter model and weighted $U$-statistics. Ann. Appl. Prob. 7, 1080-1105.

[26] Stein, C. (1972). A bound for the error in the normal approximation to the distribution of a sum of dependent random variables. In Proc. Sixth Berkeley Symp. Math. Statist. Prob. (Berkeley, CA, 1970/1971), Vol. 2, University of California Press, pp. 583-602.

[27] Stein, C. (1972). Estimation of the mean of a multivariate normal distribution. Ann. Prob. 9, 1135-1151.

[28] Stein, C. (1986). Approximate Computation of Expectations (Inst. Math. Statist. Lecture Notes Monogr. Ser. 7). IMS, Hayward, CA.

[29] Von BAHR, B. (1976). Remainder term estimate in a combinatorial limit theorem. Z. Wahrscheinlichkeitsth. 35, 131-139. 